\title{
Rechtswissenschaft als Gerechtigkeitswissenschaft
}

\author{
Josef Franz Lindner*
}

A. Einleitung: Orientierungskrise der

Rechtswissenschaft?....

B. Rechtswissenschaftstheorie?..........

C. Gerechtigkeit als Proprium der Rechtswissenschaft. ...

I. Der programmatische Auftrag: was ist gerechtes Recht?

II. Plädoyer für eine „Renaissance“ dieses Auftrags

D. Das erkenntnistheoretische Problem. .

I. Das „Münchhausentrilemma“.......

II. Plausibilität statt Substanzontologie

E. These: Die „Condicio humana“ als Erkenntnisquelle der Gerechtigkeit ...

I. Dem Menschen gemäßes Recht: Paradigma einer anthropologischen Rechtsphilosophie....

II. Zur Tradition einer anthropologischen Rechtsphilosophie.

4

7

9

9

F. Das Argumentationsprogramm einer anthropologischen Rechtsphilosophie.

I. „Naturrechtsverdacht“. ...

II. Methodische Anforderungen an die Annahme von Plausibilität

1. Die Konvergenztheorie der Wahrheit....
2. Die Kategorie des transzendentalen Interesses

III. Die „condicio humana“ als Erkenntnisquelle gerechten Rechts......... 16

1. Das Existenzielle................ 16

2. Das Substanzielle............... 17

3. Das Soziale .................... 17

4. Das Innerliche .................. 18

G. Mögliche Konsequenzen für die

Rechtswissenschaft.................. 19

I. Rechtsphilosophie: Die Begründung gerechter Rechtsinhalte............ 19

II. Rechtspolitik: Die Umsetzung gerechter Rechtsinhalte .............

III. Rechtsdogmatik: Entwicklung einer gerechtigkeitsoptimierenden Anwendungslehre ........................ 20

1. Aufgabe der Dogmatik.......... 20

2. Rechtsbereichsübergreifende Dogmatik: die Figur des Interessendreiecks ................... 23

IV. Rechtstheorie: Rechtskritik als Aufgabe ........................... 24

1. Begleitende kritische Analyse ... 25

2. Ideologiekritik ................ 25

H. Ausblick: Selbstand und Interdisziplinarität....

Die Rechtswissenschaft befindet sich in einer Orientierungskrise. Indiz: sie befasst sich in grundsätzlicher Hinsicht mit sich selbst. Dies allerdings zu recht, befindet sich doch das Konzert der normativen Steuerungsautoritäten in einem fundamentalen Wettbewerb um die Deutungshoheit: Rechtswissenschaft, Ökonomie, Politikwissenschaft, Kognitionswissenschaften sowie die Ethik und andere streiten um ihre Relevanz im normativen (und damit letztlich politisch-medialen) Diskurs. Es erstaunt daher, dass sich die Rechtswissenschaft in diesem fundamentalen Prozess sehr stark auf die formal-theoretische Dimension zurückzuziehen scheint. Dies zeigt sich exemplarisch anhand der aktuellen Diskussion um eine sog. „Rechtswissenschaftstheorie“. Nachfolgender Beitrag versteht sich als Kontrapunkt: er geht davon aus, dass die Rechtswissenschaft dauerhaft nur ernst genommen wird, wenn sie den materiellen Steuerungsanspruch des Rechts aufrecht erhält. Davon ausgehend wird für einen stärker rechtsphilosophischen Blickwinkel plädiert. Es soll die Frage erörtert

* Der Autor ist apl. Professor für Öffentliches Recht, Europarecht, Verwaltungslehre und Rechtsphilosophie an der LMU München sowie Ministerialrat im Bayerischen Staatsministerium für Wissenschaft, Forschung und Kunst. 
werden, ob sich aus der spezifisch menschlichen Bedingtheit („condicio humana“) inhaltlich richtiges (und damit sinnvoller Weise verbindliches) Recht legitimieren lässt - und was daraus für die Jurisprudenz als Wissenschaft folgen könnte.

\section{A. Einleitung: Orientierungskrise der Rechtswissenschaft?}

Es sind einige Indizien für eine (erneute) ${ }^{1}$ Orientierungsphase oder womöglich Orientierungskrise in der Rechtswissenschaft zu erkennen: Da ist zunächst die zunehmend beklagte Sprachlosigkeit zwischen den klassischen Rechtsgebieten. ${ }^{2}$ Zivilrechtler, Straf- und Öffentlich-Rechtler bleiben weitgehend jeweils unter sich. ${ }^{3}$ Eine gebietsübergreifende Diskussion findet nur vereinzelt statt. ${ }^{4}$ Hinzukommt die fortschreitende gebiets, ,interne “ Zersplitterung in verschiedene Sub-Rechtsgebiete (im Bereich des Öffentlichen Rechts etwa das Vergabe-, Regulierungs-, Telekommunikations-, Planungs-, Umwelt-, Energie-, Technikrecht etc.), die kaum verbunden nebeneinander stehen. Man kann durchaus von der Tendenz einer „Atomisierung“ des Rechts und damit der Rechtswissenschaft sprechen. ${ }^{5}$ Gemeinsame theoretische oder dogmatische Klammern sind nur vereinzelt wahrnehmbar. Verstärkt wird diese Tendenz durch die rückläufige Bedeutung ${ }^{6}$ der sog. Grundlagenfächer. ${ }^{7}$ Quasi kompen-

1 Die Befassung mit der Wissenschaftstheorie und wissenschaftstheoretische Selbstzweifel haben in der Rechtswissenschaft Tradition; vgl. dazu U. Neumann, Wissenschaftstheorie der Rechtswissenschaft, in: A. Kaufmann/W. Hassemer/U. Neumann (Hrsg.), Einführung in die Rechtsphilosophie und Rechtstheorie der Gegenwart, 7. Aufl. Heidelberg 2004, S. 385: „Die Frage..., ob und in welchem Sinne die Rechtswissenschaft eine Wissenschaft sei, beschäftigte schon im 16. Jahrhundert Philosophen und Juristen."

2 M. Jestaedt/O. Lepsius (Hrsg.), Rechtswissenschaftstheorie, Tübingen 2008, Vorwort, S. V.

3 M. Jestaedt, Perspektiven der Rechtswissenschaftstheorie, in: ders./O. Lepsius (Fn. 2), S. 205: „... Belebung des Gesprächs zwischen den 'Säulen' der Jurisprudenz, deren Vertreter sich vielleicht allzu sehr daran gewöhnt haben, ibren Horizont auf den eigenen rechtsdogmatischen 'Beritt' zu begrenzen."

4 Allerdings gibt es in jüngster Zeit gewichtige Ausnahmen: Zu nennen sind neben dem in Fn. 2 erwähnten Band vor allem C. Engel/W. Schön (Hrsg.), Das Proprium der Rechtswissenschaft, Tübingen 2007, wo die Frage, was den Wissenschaftscharakter der Rechtswissenschaft ausmache, aus der Sicht des Zivil-, Straf- und Öffentlichen Rechts behandelt wird. Auch die durch die moderne Hirnforschung neu belebte Diskussion über die Willensfreiheit und ihre Bedeutung für das Recht wird bereichsübergreifend geführt, vgl. etwa den Sammelband von E.-J. Lampe/M. Pauen/G. Roth (Hrsg.), Willensfreiheit und rechtliche Ordnung, Frankfurt a.M. 2008, wo die Thematik u.a. aus der Perspektive des Zivilrechts, des Strafrechts und des Staatsrechts beleuchtet wird.

5 Deutlich H. Schulze-Fielitz (Hrsg.), Staatsrechtslehre als Wissenschaft, Berlin 2007, S. 35: „Diversifizierung “ und individuelle Spezialisierungsprozesse als „Megatrend“ mit der Folge, „dass der Blick auf den Zusammenhang der verschiedenen Fächer ... verloren zu gehen droht."

6 Ob dies auch auf die Drittmittelorientiertheit der Hochschulpraxis einerseits und auf die damit verbundene jüngere Herausbildung von speziellen Lehrstuhldenominationen andererseits zurückzuführen ist, sollte Gegenstand eines eigenen rechtsempirischen Forschungsprojekts sein.

7 Dazu etwa E. Hilgendorf, Zur Lage der juristischen Grundlagenforschung in Deutschland heute, in: W. Brugger/U. Neumann/S. Kirste (Hrsg.), Rechtsphilosophie im 21. Jahrhundert, Frankfurt a.M. 2008, S. 111; deutlich A. Somek, Rechtliches Wissen, Frankfurt a.M. 2006, S. 10 im Hinblick auf die Rechtstheorie: „.. ist die Rechtstheorie sozial erkennbar daran, dass sie von juristischen Experten als für das handfeste juristische Geschäft entbehrlich erlebt wird. " 
satorisch orientiert man sich an den Sozialwissenschaften, ${ }^{8}$ zumal der Systemtheorie, ${ }^{9}$ also an grundsätzlich eher empirisch und beschreibend denn normativ arbeitenden Disziplinen. Die materiell-normative Steuerungsfunktion des Rechts hingegen wird tendenziell vernachlässigt zu Gunsten der Etablierung eines funktional-technokratischen ${ }^{10}$ Beschreibungssystems. ${ }^{11}$ Die Rechtswissenschaft droht somit - jedenfalls im Bereich der Wissenschaft vom Öffentlichen Recht ${ }^{12}$ - ihren wissenschaftlichen Selbstand als Normwissenschaft zu verlieren, und damit ihren „Markenkern“.

Diese Entwicklung ist insofern prekär, als die Herausforderungen, die das Recht und damit auch die Rechtswissenschaft zu verarbeiten haben, größer werden. Einige Beispiele seien herausgegriffen: ${ }^{13}$ Die Internationalisierung und Europäisierung des Rechts bedürfen nicht nur der theoretisch ordnenden Systematik und praxisbezogenen Dogmatik, sie verlangen auch materiell nach Bewahrung rechtsstaatlicher, de-

8 Diese Tendenzen finden sich insbesondere im Bereich der sog. „Governance“-Forschung und manchen Beiträgen zur sog. „neuen Verwaltungsrechtswissenschaft“; vgl. etwa G.F. Schuppert, Verwaltungsrecht und Verwaltungswissenschaft im Wandel. Von Planung über Steuerung zu Governance? AöR 133 (2008), S. 79 m.w.N.; C. Franzius, Funktionen des Verwaltungsrechts im Steuerungsparadigma der Neuen Verwaltungsrechtswissenschaft, DV 2006, S. 335; A. Voßkuble, Neue Verwaltungsrechtswissenschaften, in: W. Hoffmann-Riem/E. Schmidt-Aßmann/A. Voßkuhle (Hrsg.), Grundlagen des Verwaltungsrechts I, München 2006, $\mathbb{S} 1$.

9 Dazu und zu den Konsequenzen frühzeitig kritisch O. Lepsius, Steuerungsdiskussion, Systemtheorie und Parlamentarismuskritik, Tübingen 1999, S. 7: „Es besteht die Gefahr, dass die Ordnungsvorstellung des Verfassungsrechts durch Ordnungsvorstellungen der Makrosoziologie ersetzt werden und es dadurch zu einem Autonomieverlust der Rechtswissenschaft, aber auch zu einer Relativierung der normativen Kraft der Verfassung kommt."

10 Vgl. T. Raiser, Grundlagen der Rechtssoziologie, 4. Aufl. Tübingen 2006, S. 155, wo der Zusammenhang von Systemtheorie und transindividueller Technokratie klar gesehen wird: „Während diese (i.e. die systemtheoretischen Ansätze der Soziologie, J.F.L.) gerade die Ein- und Unterordnung des Menschen unter die intermediären Gewalten herausarbeiten ... und dabei leicht in ein technokratisches Rechtsdenken ausmünden ...".

11 Prägnant P.M. Huber, Die Demontage des Öffentlichen Rechts, in: W. Kluth/M. Müller/A. Peilert (Hrsg.), Festschrift für Stober, Köln 2008, S. 547 (556): „Dabei werden die höchstkomplexen Realbefunde, organisationssoziologische, entscheidungstheoretische und politikwissenschaftliche Einsichten ... häufig nach Art einer wirklichkeitswissenschaftlichen Betrachtung zum Nennwert genommen und die Rechtsauslegung und -anwendung fälschlicherweise daran ausgerichtet. Hier erst beginnt jedoch die eigentliche Aufgabe des Öffentlichen Rechts. Realbefunde ... müssen an die normativen Steuerungsvorgaben angebunden und in rechtlich belastbare... Handlungsformen, Institute und Verfahren umgesetzt werden."

12 Von daher - aber auch wegen des engen Politik- und damit Machtbezugs - ist es nicht verwunderlich, dass die Orientierungsdiskussion gerade im Öffentlichen Recht auf Hochtouren läuft. Die Themen der Staatsrechtslehrertagungen der letzten Jahre (2007: „Die Leistungsfähigkeit der Wissenschaft des Öffentlichen Rechts“; 2008: „Erosionen der Verfassungsvoraussetzungen“) legen davon ebenso beredt Zeugnis ab wie der Sammelband von H. Schulze-Fielitz (Hrsg.), Staatsrechtslehre als Wissenschaft, Berlin 2007.

13 Zu nennen wären ferner die infolge von Umwelt- und Klimaveränderungen, zumal von Wasserknappheit und Hungersnöten drohenden Migrationsbewegungen und deren zivilisatorische Bewältigung, die Bändigung der Diktatur des Ökonomischen sowie die friedensorientierte Steuerung der religiös/weltanschaulichen Pluralität in der Gesellschaft. 
mokratischer und grundrechtlicher Standards. ${ }^{14}$ Der Fortschritt zumal in der Medizin und in den Bio- und Neurowissenschaften stellt unser auf der Menschenwürde basierendes Menschenbild am Anfang, bei der Gestaltung ${ }^{15}$ und am Ende des Lebens auf den Prüfstand. Die demographische Entwicklung verlangt nach Veränderungen auch in der Rechtsordnung. Welchen „Steuerungsanspruch“ wird in Anbetracht dieser Herausforderungen das Recht im Konzert der Steuerungsautoritäten (insbes. der Parteien, der Ökonomie und der Kognitionswissenschaften) haben (bzw. behalten) und wie wirkt sich dies auf den Anspruch der Rechtswissenschaft als Normwissenschaft aus?

Zu solchen Fragen ist in jüngster Zeit eine verstärkte Grundlagendiskussion auszumachen. Dabei stehen allerdings eher rechts- und wissenschaftstheoretische Ansätze im Vordergrund (B.). Weniger intensiv werden die Grundlagen der Rechtswissenschaft aus materiell-rechtsphilosophischer Sicht erörtert. Der Frage nach dem inhaltlich richtigen, dem gerechten Recht sollte stärker nachgegangen werden (C.). Das ist wegen der unvermeidbaren Begründungsprobleme allerdings methodisch prekär, insbesondere aufgrund der heute kaum mehr bestrittenen Unmöglichkeit von metaphysischen Wahrheiten oder substanzontologischen Letztbegründungen (D.). Man muss sich daher mit einem weniger ambitionierten Weg begnügen. Ein solcher könnte darin bestehen, materielle Inhalte des Rechts und damit ein gerechtigkeitsfundiertes Orientierungsprogramm für die Rechtswissenschaft aus der natürlichen Bedingtheit des Menschen, der „condicio humana“ herzuleiten (E.). Wegen seines Naturrechts, verdachts“ muss das Argumentationsprogramm eines solchen rechtsanthropologischen Ansatzes zunächst methodisch und inhaltlich geklärt werden (F.). Sodann ist zu überlegen, welche Konsequenzen Erkenntnisse einer anthropologischen Rechtsphilosophie für die Rechtswissenschaft (G.) und ihren Status im Konzert der Wissenschaften haben könnten (H.).

\section{B. Rechtswissenschaftstheorie?}

Die Befassung mit den Grundlagen des Rechts hat jüngst an Dynamik gewonnen. Im Mittelpunkt ${ }^{16}$ stehen Fragen nach der Wissenschaftlichkeit, dem Wesen der Rechts-

14 Ein spektakuläres Beispiel ist die Rechtsprechung des EuGH zum Grundrechtsschutz gegen EURechtsakte, die der Umsetzung von Maßnahmen der Vereinten Nationen zur Bekämpfung des internationalen Terrorismus dienen, insbesondere das „Einfrieren“ von Geldern; EuGH NJW 2008, S. 3697.

15 Vgl. etwa J.F. Lindner, „Neuro-Enhancement“ als Grundrechtsproblem., MedR 2010, S. 463.

16 Insbesondere die Rechtstheorie erfährt neue Belebung, auch wenn sich insoweit ein inhaltlicher Schwerpunkt kaum ausmachen lässt; vgl. etwa die Vielfalt indes nicht durchweg „neuer“ Theorien des Rechts bei S. Buckel/R. Christensen/A. Fischer-Lescano (Hrsg.), Neue Theorien des Rechts, Stuttgart 2006; s. ferner den rezensierenden Überblick bei B. Lahusen/D. Simon, Zufall, Abfall, Ausfall Rezensionen und Betrachtungen zur rechtstheoretischen Gegenwartsliteratur, Frankfurt a.M. 2008. $\mathrm{Zu}$ „Ort und Funktion“ der Rechtstheorie s. T. Vesting, Rechtstheorie, München 2007, S. 1 ff. Zu einer rhetorischen Evolution des Rechts s. F. Steinhauer, Gerechtigkeit als Zufall, Wien, NewYork 2007. 
wissenschaft. Eine neue Schriftenreihe steht unter dem Motto: „Recht - Wissenschaft - Theorie. Standpunkte und Debatten“. ${ }^{17}$ Ziel dieser Reihe ist es, den „Theoriebezug der Rechtswissenschaft in den Mittelpunkt“ zu rücken, es geht um „das Proprium der Rechtswissenschaft als einer theoretisch angeleiteten und nicht bloß praktisch motivierten Wissenschaft“. In der bisherigen Debatte, die sich in den bislang erschienenen Bänden dieser Reihe ${ }^{18}$ widerspiegelt, überwiegt der wissenschaftstheoretische Problemdiskurs. Namentlich der zweite Band thematisiert die Frage, was „Rechtswissenschaftstheorie“ - ein Ausdruck, der bisher so nicht verwandt wurde ${ }^{19}$ -, sei und wozu sie diene. Unter „Rechtswissenschaftstheorie “20 wird - bei aller Abgrenzungsproblematik etwa zur Rechtstheorie oder zur Methodenlehre ${ }^{21}$ - eine Art „Metatheorie“22 oder „Meta-Disziplin der Jurisprudenz“23 verstanden, die sich mit den Bedingungen der Wissenschaftlichkeit der Rechtswissenschaft und auf dieser Basis mit dem „Verhältnis der einzelnen juridischen (Sub-)Disziplinen “ 24 zu einander befasst. Ein weiterer Forschungsschwerpunkt einer Rechtswissenschaftstheorie liegt in der Erörterung der Bedingungen interdisziplinärer Zusammenarbeit mit anderen Disziplinen, den sog. „Nachbardisziplinen“ der Rechtswissenschaft (Soziologie, Politische Wissenschaften, Wirtschaftswissenschaften, Psychologie ${ }^{25}$ etc.). Dies ist wichtig, weil es für Akzeptanz und Gelingen interdisziplinärer Forschungsprojekte unabdingbar ist, methodische Instrumente als „Metaregeln interdisziplinärer Zusammenarbeit " ${ }^{26} \mathrm{zu}$ formulieren und dadurch den Selbstand der Rechtswissenschaft

17 Herausgegeben von M. Jestaedt/O. Lepsius/C. Möllers/A. Voßkuble.

18 Band 1: C. Engel/W. Schön (Hrsg.), Das Proprium der Rechtswissenschaft, Tübingen 2007 (vgl. dazu bereits Fn. 4); Band 2: M. Jestaedt/O. Lepsius (Hrsg.), Rechtswissenschaftstheorie, Tübingen 2008 (Fn. 2); Band 3: A. Funke/J. Lüdemann (Hrsg.), Öffentliches Recht und Wissenschaftstheorie, Tübingen 2009.

19 Jestaedt, Rechtswissenschaftstheorie (Fn. 3), S. 186: „noch unbesetzt“. Man sprach bislang eher von „Wissenschaftstheorie der Rechtswissenschaft" (vgl. oben Fn. 1).

20 Zur Systematisierung der möglichen Begriffsverständnisse s. Jestaedt, Rechtswissenschaftstheorie (Fn. 3), S. 186.

$21 \mathrm{Zu}$ diesen Abgrenzungen s. C. Möllers, Vorüberlegungen zu einer Wissenschaftstheorie des öffentlichen Rechts, in: M. Jestaedt/O. Lepsius (Fn. 18), S. 156 sowie M. Jahn, Pluralität der Rechtsdiskurse, in: M. Jestaedt/O. Lepsius (Fn. 18), S. 175.

22 Teilweise ist auch von „Mesotheorie“ die Rede, so bei A. $v$. Aaken, Funktionale Rechtswissenschaftstheorie für die gesamte Rechtswissenschaft, in: M. Jestaedt/O. Lepsius (Fn. 18), S. $81 \mathrm{f}$.

23 Jestaedt, Rechtswissenschaftstheorie (Fn. 3), S. 201.

24 Jestaedt/Lepsius, Rechtswissenschaftstheorie (Fn. 18), Vorwort S. V.

25 Die Rechtspsychologie ist in Deutschland allerdings faktisch „tot“. Dies ist umso erstaunlicher, als in der psychologischen Analyse des Verhaltens der Rechtsakteure nicht nur in der gerichtlichen Praxis ein Schlüssel zum Verständnis des Rechts und seiner Entstehung wie Anwendung liegen dürfte. Auch im Rahmen der sich herausbildenden Forschungsrichtung „behavioral law and economics“ dürfte auf psychologischen (und sozialwissenschaftlichen) Sachverstand nicht zu verzichten sein; H. Wieduwilt, Juristen auf den Spuren der Unvernunft - Rechtswissenschaft öffnet sich der Verhaltensforschung, FAZ v. 6.1.2009, S. 12; H. Eidenmüller, Der homo oeconomicus und das Schuldrecht: Herausforderungen durch Behaviour Law and Economics, JZ 2005, S. 216. In der Zusammenarbeit von Rechtswissenschaft und Psychologie dürften ertragreiche Erkenntnisse über das Funktionieren rechtlicher Steuerungsmechanismen „schlummern“.

26 So eine treffende Formulierung von Voßkuble, Verwaltungsrechtswissenschaften (Fn. 8), Rn. 39. 


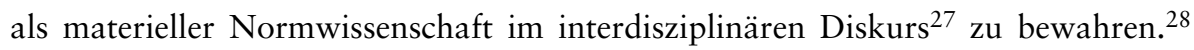
Der Begriff „Rechtswissenschaftstheorie“ ist anschlussfähig für die Frage, unter welchen Bedingungen Recht „richtig“, das heißt „gerecht“ ist. Gleichwohl überwiegt bislang der theoretische Zugriff, die „Rechtswissenschaftstheorie“ hat die (materielle) Rechtsphilosophie noch nicht erreicht. Wenn aber zutreffend betont wird, dass Rechtswissenschaftstheorie „auch als normative konzipiert sein" 29 muss, lässt sich die Frage nach den materiellen Grundlagen des Normativen mit einiger Berechtigung stellen.

\section{Gerechtigkeit als Proprium der Rechtswissenschaft}

\section{Der programmatische Auftrag: was ist gerechtes Recht?}

Auch im Bereich der Rechtsphilosophie sind wieder ${ }^{30}$ intensivere Diskussionen zu beobachten. ${ }^{31}$ Ein jüngst erschienener Sammelband „Rechtsphilosophie im 21. Jahrhundert“32 hat sich zum Ziel gesetzt, angesichts neuer Herausforderungen „eigenständige Analysen, Begriffsbildungen und Theorieansätze“ vorzustellen. ${ }^{33}$ Grundsätzlich geht es der Rechtsphilosophie um zwei Fragen: ${ }^{34}$ Erstens: Muss Recht gerecht sein? Zweitens: Wie kann gerechtes Recht begründet werden? Die Rechtsphilosophie hat es mithin in erster Linie mit der Gerechtigkeit zu tun. Sie legt die materiellen Grundlagen der Rechtswissenschaft als Gerechtigkeitswissenschaft. Die Verwirklichung von Gerechtigkeit ist der Auftrag des Rechts und damit das Proprium der Wissenschaft vom Recht.

27 Vgl. für die „Zusammenarbeit“ zwischen Ökonomik und öffentlichem Recht J.F. Lindner, Verfassungsrechtliche Rahmenbedingungen einer ökonomischen Theorie des Öffentlichen Rechts, JZ 2008, S. 957 (960).

28 Zutreffend $v$. Aaken, Rechtswissenschaftstheorie (Fn. 22), S. 88: „Eine Rechtswissenschaftstheorie sollte wissenschaftstheoretisch streng zwischen sozialwissenschaftlichen Tatsachenaussagen (Beschreibung, Erklärung und Prognose) und rechtspolitischen Wertaussagen trennen."

29 Jestaedt, Rechtswissenschaftstheorie (Fn. 3), S. 205.

30 Für eine Bestandsaufnahme s. A. Kaufmann, Problemgeschichte der Rechtsphilosophie, in: ders./W. Hassemer/U. Neumann (Fn. 1), S. 26; ders., Rechtsphilosophie, 2. Aufl. München 1997; U. Neumann, Rechtsphilosophie in Deutschland seit 1945, in: D. Simon (Hrsg.), Rechtswissenschaft in der Bonner Republik, Frankfurt a.M. 1994, S. 145; A. Brockmöller/E. Hilgendorf (Hrsg.), Rechtsphilosophie im 20. Jahrhundert - 100 Jahre Archiv für Rechts- und Sozialphilosophie, Stuttgart 2009.

31 Vgl. etwa T. Osterkamp, Juristische Gerechtigkeit, Tübingen 2004; J. Schapp, Freiheit, Moral und Recht. Grundzüge einer Philosophie des Rechts, Tübingen 2005; D. von der Pfordten, Normativer Individualismus und das Recht, JZ 2005, S. 1069; ders., Was ist und wozu Rechtsphilosophie? JZ 2004, S. 157; ders., Was ist Recht? Ziele und Mittel, JZ 2008, S. 641; H. Otto, Diskurs über Gerechtigkeit, Menschenwürde und Menschenrechte, JZ 2005, S. 473.

32 Herausgegeben 2008 von W. Brugger/U. Neumann/S. Kirste (Fn. 7).

33 Vorwort S. 7; vgl. die im Hinblick auf diesen hohen Anspruch eher skeptische Rezension bei M. Pawlik, FAZ v. 5.1.2009, S. 6: „Der Gesamteindruck ist zwiespältig. Die Disziplin Rechtsphilosophie funktioniert, daran ist kein Zweifel. ... Was aber weitgehend fehlt, ist das Innovative, Verblüffende, Provozierende."

34 Vgl. Kaufmann, Rechtsphilosophie (Fn. 30), S. 9. 


\section{Plädoyer für eine „Renaissance“ dieses Auftrags}

Allerdings steht - insbesondere in den sog. prozeduralen Gerechtigkeitsdiskursen ${ }^{35}$ - die materiell-rechtsphilosophische Frage nach dem richtigen, gerechten Recht derzeit weniger im Fokus. ${ }^{36}$ Es lassen sich einige Gründe dafür anführen, die materielle Gerechtigkeitsfrage wieder stärker in den Mittelpunkt der rechtswissenschaftlichen Grundlagendebatte zu rücken: ${ }^{37}$

- Zunächst verlangen die inhaltlichen Herausforderungen für Recht und Rechtswissenschaft (oben A.) inhaltliche Antworten, über deren Richtigkeit es der rechtsphilosophischen Vergewisserung bedarf.

- Die Suche nach der Gerechtigkeit kann eine einheitsstiftende Funktion für die Rechtswissenschaft als wissenschaftliche Disziplin insgesamt haben. Jede Wissenschaft braucht einen Kristallisationspunkt, von dem alles „Streben“ ausgeht und auf den sich Erkenntnisse, Thesen, Antithesen und deren Falsifikation zurückbeziehen lassen. In der Medizin ist es der Mensch, in den Religionswissenschaften Gott - in der Rechtswissenschaft ist es die Gerechtigkeit. In Anlehnung an Gustav Radbruch: Recht ist das Phänomen, das den Zweck hat, der Gerechtigkeit zu dienen. ${ }^{38}$ Bei aller Komplexität, Technizität und Diversivität unserer europäisch und zunehmend international vernetzten Rechtsordnung(en) kann der Dienst an der Gerechtigkeit, das Ringen um sie im Rechtsalltag sinnstiftendes und verbindendes Element sein.

- Eine Reflexion der materiellen Legitimationsgrundlagen des Rechts könnte der Rechtswissenschaft zudem ein stärkeres Maß an Zeitgeistresistenz vermitteln und sie (wieder) stärker als kritische Wissenschaft etablieren. Eine materiell fundierte

35 Vgl. dazu unten D.II.

36 Es gibt freilich gewichtige Ausnahmen: vgl. z.B. E. Nass, Der humangerechte Sozialstaat, Tübingen 2006; O. Höffe, Politische Gerechtigkeit. Grundlegung einer kritischen Philosophie von Recht und Staat, Frankfurt a.M. 1987; ders., Kategorische Rechtsprinzipien, Frankfurt a.M. 1995; W. Kersting, Theorien der sozialen Gerechtigkeit, Stuttgart 2000; vgl. jüngst auch G. Sprenger, Von der Wahrheit zum Wert. Gedanken zu Recht und Gerechtigkeit, Stuttgart 2010.

37 Mit geradezu beschwörenden Worten A. Kaufmann, 45 Jahre erlebte Rechtsphilosophie, in: R. Alexy/ R. Dreier/U. Neumann (Hrsg.), Rechts- und Sozialphilosophie in Deutschland heute, ARSP (Beih. 44), 1991, S. 161: „Die Rechtsphilosophie muss sich mehr auf die Inhalte des Rechts besinnen. Ihre Aufgabe ist es, der Gerechtigkeit einen Weg zu bahnen. Mit Theorien, die auf sterile Reinheit bedacht sind, ist das nicht zu machen. Der Weg ist steinig und schmutzig, und wenn man ihn geht, holt man sich Blessuren. Aber was wäre die Alternative? " 
Theorie der Rechtskritik ${ }^{39}$ könnte auch der jüngst geforderten Lösung der Rechtswissenschaft von der Fixierung auf die Dogmatik (verstanden im Sinne bloßer Anwendungsdogmatik) Rechnung tragen. ${ }^{40}$

- Des Weiteren könnte eine gerechtigkeitsorientierte Rechtswissenschaft einen substanziellen Beitrag zur Stabilität der konkreten Rechtsordnung in der Rechtspraxis und im Ansehen der Bevölkerung leisten. ${ }^{41}$ Da Recht mit Androhung und Anwendung von Zwang verbunden ist, fragt der davon betroffene Mensch - wenn nicht immer bewusst, aber doch intuitiv - nach der Legitimation dafür. Jeder rechtliche Zwang ist aus der Sicht des betroffenen Menschen legitimationsbedürftig. Zur Rechtfertigung dürfte es regelmäßig nicht allein genügen, vorzutragen, die entsprechende Regelung sei demokratisch und damit formal ordnungsgemäß zu Stande gekommen. Es bedarf vielmehr der Beantwortung der Frage, ob die Zwangsausübung gerecht ist. Diese Frage kann formal und demokratietheoretisch nicht hinreichend beantwortet werden, notwendig sind vielmehr materielle, von den „rechtsunterworfenen“ Menschen akzeptierte oder zumindest (intersubjektiv) akzeptierbare Maßstäbe. Durch eine - durchaus auch populärwissenschaftlich engagierte - Vermittlung der materiell legitimierbaren Grundlagen der Rechtsordnung und der Rückbezüge der Einzelrechtsgebiete und juristischen Einzelrechtsfragen auf diese Grundlagen könnten bei Rechtsbetroffenen Verständnis und letztlich Akzeptanz zumindest gefördert werden.

- Schließlich könnte die Reflexion des Gerechtigkeitsbezugs den Selbstand der Rechtswissenschaft als Normwissenschaft stärken - in normativ geprägter Abgrenzung zu den empirischen Sozialwissenschaften. Das heißt nicht Verweigerung des interdisziplinären Diskurses, sondern Schärfung der spezifisch normativen Rolle der Rechtswissenschaft als Gerechtigkeitswissenschaft ${ }^{42}$ in diesem Diskurs (s. auch unten H.).

39 Es sind verschiedene Modalitäten, Stufen der Rechtskritik zu unterscheiden: (1) Dogmatische Rechtskritik, bei der die Kritik dogmatischer Begriffe, Figuren und Argumentationsschemata im Mittelpunkt steht - das ist das "Alltagsgeschäft" wissenschaftlich geleiteter Rechtsdogmatik. (2) Strukturelle Rechtskritik, die sich auf bestimmte Strukturen eines Rechtsgebietes bezieht, insbesondere Wertungswidersprüche, strukturelle Brüche oder inhaltliche Inkohärenzen im Blick hat. (3) Ontologische Rechtskritik, die das Recht und seine Erscheinungsformen in fundamentaler, zumal materieller Hinsicht hinterfragen oder gar in Frage stellt. Zu nennen sind v.a. der Dekonstruktivismus (Derrida) und Denkrichtungen, die den - in Deutschland wenig wahrgenommenen - „critical legal studies“ zuzurechnen sind, vgl. dazu G. Frankenberg, Partisanen der Rechtskritik, in: Buckel/Christensen/FischerLescano, Rechtstheorie (Fn. 16), S. 97.

40 O. Lepsius, Themen einer Rechtswissenschaftstheorie, in: Jestaedt/Lepsius, Rechtswissenschaftstheorie (Fn. 18), S. 20.

41 Vgl. jüngst R. Forst, Das Recht auf Rechtfertigung. Elemente einer konstruktivistischen Theorie der Gerechtigkeit, Frankfurt a.M. 2007, der das Recht des Menschen, keinen Normen unterworfen zu werden, die ihm gegenüber nicht gerechtfertigt werden können, als „einziges grundlegendes Recht des Menschen" qualifiziert.

42 Speziell für das Öffentliche Recht J.F. Lindner, „Gewissheit“ als Paradigma der Wissenschaft vom Öffentlichen Recht - Versuch einer Grundlegung aus rechtsphilosophischer Sicht, JöR n.F. 57, Tübingen 2009, S. 173 (205). 


\section{Das erkenntnistheoretische Problem}

\section{Das „Münchhausentrilemma“}

Der zu erwartende Einwand gegen die These, Rechtswissenschaft müsse Gerechtigkeitswissenschaft sein, liegt nicht fern: Gerechtigkeitsmaßstäbe als dem Recht voraus oder ihm inne liegende Substanz ließen sich nicht allgemein- und letztverbindlich begründen, seien in ihrem Inhalt willkürlich und damit kein geeigneter Reflexionsgegenstand für die Rechtswissenschaft. Auch nach mehr als zweitausend Jahren Ethik und (Rechts-)Philosophie gebe es keine Einigkeit über den Inhalt der Gerechtigkeit. Allenfalls über die Verfahren zu deren Ermittlung lasse sich auf Grund der Diskurs- und Demokratietheorie einigermaßen Zuverlässiges sagen. In der Tat: die Suche nach dem gerechten Recht und einer darauf bezogenen Rechtswissenschaft droht in einer Aporie zu enden, die Hans Albert plastisch als „Münchhausentrilemma“43 bezeichnet hat. Der Versuch der Begründung normativer Inhalte als gerecht und das daraus abgeleitete Postulat, solche Inhalte müssten auch Rechtsinhalte sein, müsse entweder in einen infiniten Regress (etwa: warum ist ein bestimmter normativer Inhalt gerecht? warum ist der Grund dafür stichhaltig? u.s.w.), in einen logischen Zirkel (etwa: der normative Inhalt X ist gerecht, weil er dem Wesen des Menschen entspricht und gerecht ist alles, was dem Wesen des Menschen entspricht) oder in den Abbruch des Begründungsverfahrens an einer bestimmten Stelle münden. Alle drei „Lösungen“ sind im Hinblick auf eine Letztbegründung der Richtigkeit normativer Inhalte ${ }^{44}$ nicht befriedigend. Naturrechtliche oder rechtsethische Postulate sind nicht letztbegründbar, aus der Moral folgt weder logisch noch normativ zwingend etwas für das Recht. ${ }^{45}$

\section{Plausibilität statt Substanzontologie}

Daraus ist aber nicht zwingend zu schließen, dass es zwischen dem Recht und außerrechtlichen Legitimationsmaßstäben überhaupt keine normativen Verbindungen ge-

43 H. Albert, Traktat über kritische Vernunft, 5. Aufl. Tübingen 1991, S. 9.

44 Für die Moralphilosophie jüngst N. Hoerster, Was ist Moral? München 2008. Grundsätzlich zum Problem der Begründbarkeit moralischer Inhalte D. Birnbacher, Analytische Einführung in die Ethik, Berlin, New York, 2003, insbes. S. 354; P. Stemmer, Handeln zugunsten anderer - eine moralphilosophische Untersuchung, Berlin, New York 2000 sowie J.L. Mackie, Ethik. Die Erfindung des moralisch Richtigen und Falschen, Stuttgart 1981.

45 Die in verschiedenen Modalitäten (Recht und Gerechtigkeit, Recht und Moral, Recht und Ethik, Rechtsethik; vgl. D. v.d. Pfordten, Rechtsethik, München 2001; ders., Normative Ethik, Berlin 2010) gestellte Frage, unter welchen Voraussetzungen positives Recht richtiges Recht ist und welche außerpositivrechtlichen Maßstäbe dafür als Beurteilungskriterien dienen können, formuliert das Kernproblem der Rechtsphilosophie. Vgl. dazu H. Welzel, Naturrecht und materiale Gerechtigkeit, Heidelberg 1962; Kaufmann, Rechtsphilosophie (Fn. 30), S. 20; G. Ellscheid, Strukturen naturrechtlichen Denkens, in: A. Kaufmann/W. Hassemer/U. Neumann (Fn. 1), S. 148; N. Hoerster, Was ist Recht? München 2006, S. 92 („,ethische Anforderungen an das Recht“). 
ben könne oder dürfe, wie es die meisten ${ }^{46}$ rechtspositivistischen Trennungsmodel$1 \mathrm{e}^{47}$ annehmen. Gemeinsam dürfte allen Rechtspositivismen die Annahme sein, dass es keinen zwingenden Zusammenhang von Moral- und Rechtsinhalten gebe. Damit ist freilich nicht gesagt, dass nicht auch der - selbst in der Sache strengste - Rechtspositivist die Richtigkeit des Rechts aus moralischen oder religiösen Motiven fordern könnte. Positivismus und Wert-Relativismus gehen nicht notwendig Hand in Hand. Auch ein Rechtspositivist könnte daher eine anthropologische Rechtsphilosophie durchaus vertreten, auch wenn er ihr die normative Relevanz für den Rechtsetzer abspricht. Holzschnittartig: Der Rechtspositivist weist die Umsetzung der Erkenntnisse der Rechtsphilosophie allein der Rechtspolitik zu, der „Naturrechtler“ nimmt eine Bindung an. Davon zu unterscheiden ist die Frage, welche Konsequenzen ein Verstoß gegen eine solche Bindungswirkung für die Geltung des verstoßenden Rechtsaktes hätte. Unterstellt also, dass es keine zwingenden normativen, also naturrechtlich-substanzontologischen Verbindungen zwischen Recht und außerrechtlichen Wertmaßstäben geben kann, ist damit nicht automatisch gesagt, dass sich nicht doch Legitimationskonnexe herstellen lassen, die auf Plausibilitätserwägungen beruhen. Solche Plausibilitäten zu begründen, ist Anliegen der Begründungsstrategien zwischen dem substanzontologisch verbindlichen Naturrecht und dem normativ durch außerrechtliche Richtigkeitsmaßstäbe nicht beeinflussbaren positiven Recht. Dazu sind eine Fülle von Thesen und Theorien entwickelt worden. ${ }^{48}$ Die (wohl auch heute noch) dominierende Strömung ${ }^{49}$ sind die sog. „Prozeduralen Theorien der Gerechtigkeit". ${ }^{50}$ Diese leiten - vereinfacht - die Richtigkeit von normativen Inhalten aus ihrem Zustandekommen in einem bestimmten Verfahren ab. ${ }^{51}$ Hierher gehören neben vertraglichen Legitimationsmodellen ${ }^{52}$ wie etwa der "Theorie der Gerechtigkeit" von J. Rawls die Diskurstheorien, die von der Einhaltung von formalen Dis-

46 Maßgeblich H. Kelsen, Reine Rechtslehre, 2. Aufl., Wien 1960, will die Rechtswissenschaft von allen ihr fremden Elementen befreien. $\mathrm{Zu}$ nennen ist hier auch die analytische Rechtstheorie um H.L.A. Hart; vgl. v.a. Der Positivismus und die Trennung von Recht und Moral, in: ders. Recht und Moral, hrsg. v. N. Hoerster, Göttingen 1971, S. 14.

47 Vgl. dazu Hoerster, Recht (Fn. 45), S. 65 („Der sog. Rechtspositivismus“), der zutreffend herausstellt, dass es mehrere Spielarten des Rechtspositivismus zu unterscheiden gilt.

48 Ausführlich und mit umfangreichen Nachweisen Kaufmann, Rechtsphilosophie (Fn. 30) S. 39.

$49 \mathrm{Zu}$ weiteren - hier nicht zu vertiefenden - Theorien s. S. Buckel/R. Christensen/A. Fischer-Lescano, Rechtstheorie (Fn. 16).

50 Vgl. etwa A. Tschentscher, Prozedurale Theorien der Gerechtigkeit, Baden-Baden 2000.

51 Vgl. die Formulierung bei R. Alexy, Probleme der Diskurstheorie, Zeitschrift für philosophische Forschung 43 (1989), S. 81: „Nach allen prozeduralen Theorien hängt die Richtigkeit einer Norm oder die Wabrheit einer Aussage davon ab, ob die Norm oder die Aussage das Ergebnis einer bestimmten Prozedur ist oder sein kann."

52 Dazu grundsätzlich W. Kersting, Die politische Philosophie des Gesellschaftsvertrages, Darmstadt 1994. 
kursregeln auf die Richtigkeit des Inhaltes schließen. ${ }^{53}$ Man kann in Anlehnung an die naturrechtliche Substanzontologie von einem formalontologischen Schluss sprechen: Die Wahrung bestimmter Formen ist zumindest ein gewichtiges Indiz für die Richtigkeit des Inhalts. Die Angriffsfläche der Diskurstheorien liegt in eben diesem Schluss. A. Kaufmann fragt mit Recht: „Haben wir hier nicht wieder diese geheimnisvolle Urzeugung des Stoffes aus der Form?" 54 Anders gefragt: Kann die Einhaltung formaler Maßstäbe allein für die Annahme der Plausibilität von Inhalten genügen?

\section{E. These: Die „Condicio humana“ als Erkenntnisquelle der Gerechtigkeit}

\section{Dem Menschen gemäßes Recht: Paradigma einer anthropologischen Rechtsphilosophie}

Im Gegensatz zu den soeben angesprochenen formellen Plausibilitätsmodellen besteht der hier präferierte Weg im Versuch einer Begründung materieller Plausibilität. Die nachfolgend zu begründende Grundthese lautet: Recht ist richtig, wenn es dem Menschen in seiner natürlichen Erschaffenheit, seiner Kreatürlichkeit gerecht wird. Aus der Analyse des Menschseins in seiner tatsächlichen Beschaffenheit, seiner natürlichen Ausstattung und Bedingtheit, kurz: der "condicio humana“ lassen sich zwar nicht normativ zwingende, aber doch materiell plausible Vorgaben für die Inhalte einer gerechten Rechtsordnung ableiten. Im Gegensatz zu religiösen, materiell anthropologischen, sonst weltanschaulichen, kurz metaphysischen Menschenbildern stellt die hier vorgeschlagene Form einer anthropologischen Rechtsphilosophie den Menschen in seinem - normativ ungeprägten - gattungsdeterminierten So-Sein in den Mittelpunkt der Normlegitimation. Zu diesem So-Sein gehören jedenfalls drei Dimensionen (s. i.E. unten F.III.):

- Die existentielle Dimension: der Mensch ist realiter hineingestellt („geworfen“) in die Welt als seiendes, zunächst schutzloses, bedrohtes, auf Hilfe und Gemeinschaft, auf Schutz seiner Integrität verwiesenes Wesen.

- Die individuell-kulturelle Dimension, die die natürliche, aus der Vernunftbegabung resultierende Bestrebung des Menschen im Blick hat, sich zu entfalten, seine Ideen und Fähigkeiten selbstbestimmt einzusetzen, mit anderen zu kommunizieren, zu kooperieren und mitunter sogar über sich hinauszuwachsen.

53 Vgl. dazu J. Habermas, Theorie des kommunikativen Handelns, Frankfurt a.M. 1981; ders., Diskursethik - Notizen zu einem Begründungsprogramm, in: Moralbewusstsein und kommunikatives Handeln, Frankfurt a.M. 1983, S. 53; s. auch die Darstellung bei R. Alexy, Theorie der juristischen Argumentation, 2. Aufl. Frankfurt a.M. 1991, S. 134 und bei S. Chambers, Zur Politik des Diskurses: Riskieren wir unsere Rechte? in: K.-O. Apel/M. Kettner (Hrsg.), Zur Anwendung der Diskursethik in Politik, Recht und Wissenschaft, Frankfurt a.M. 1993, S. 168 (170).

54 A. Kaufmann, Rechtsphilosophie (Fn. 30) S. 276. 
- Schließlich die distributiv-soziale Dimension, die darauf verweist, dass der Mensch zur Verwirklichung von (1) und (2) auf materielle Ressourcen und tatsächliche Chancen angewiesen ist.

Diese Dimensionen muss eine Rechtsordnung, die den Anspruch erhebt, gerecht zu sein, adäquat berücksichtigen. Die Rechtswissenschaft muss sie theoretisch und dogmatisch ausformen sowie in der Rechtspraxis umsetzen. ${ }^{55}$ In einem solchen Ansatz treffen sich positivistische und naturrechtliche Aspekte: Positivistisch ist er insofern, als der "archimedische Punkt“ für die Legitimation von Rechtsinhalten die tatsächliche, positiv, nicht normativ festgestellte Bedingtheit des Menschseins ist. Naturrechtliche Elemente weist die Theorie dadurch auf, dass sie an diese tatsächlich-naturgegebene Bedingtheit des Menschen normative Konsequenzen im Sinne materieller Plausibilität knüpft.

\section{Zur Tradition einer anthropologischen Rechtsphilosophie}

Versuche einer normativen Anthropologie hat es in der Geschichte der Philosophie immer wieder gegeben. ${ }^{56}$ Der Mensch war immer Gegenstand des Philosophierens, allerdings stand er nicht stets als solcher im Mittelpunkt. Vielmehr wurde er lange als unselbständiger Teil eines größeren Ganzen begriffen, eingebettet in „Überbauten “; die Quellen materieller Legitimation waren: Mythos, Logos, Religion, Idealismus, Vernunftrecht (dazwischen gab es freilich anthropozentrische Intervalle wie etwa Teile der Sophistik und Phasen der Renaissance). Erst in der Überwindung der Erklärungs- und Rechtfertigungsmonopole metaphysischer Gesamtsysteme in der Aufklärung konnte es gelingen, den Menschen selbst und seine normativ vorurteilslose Bedingtheit als materielle Legitimationsquelle anzusehen. Die Theorie, aus der zunächst normativ, religiös oder sonst weltanschaulich ungeprägten (unfestgestellten) tatsächlichen „Kreatürlichkeit“ 57 des Menschen Erkenntnisse über die normative Richtigkeit von Rechtsinhalten abzuleiten, ist freilich stets eine „Mindermeinung " geblieben. Auch in der Rechtsphilosophie ${ }^{58}$ haben anthropologische Theorien bislang wenig Aufmerksamkeit gefunden. Ein eigenständiges Forschungsgebiet

55 Zur methodischen Begründung s. näher unten F.II.

56 Das kann hier nicht vertieft werden, vgl. dazu nur den gerafften Überblick bei Kaufmann, Rechtsphilosophie (Fn. 30), S. 186; O. Schwemmer, Artikel „Anthropologie“, in: J. Mittelstraß (Hrsg.), Enzyklopädie Philosophie und Wissenschaftstheorie, Bd. 1, Stuttgart 2004, S. 126; M. Scheler, Die Stellung des Menschen im Kosmos, Darmstadt 1928; A. Geblen, Der Mensch. Seine Natur und seine Stellung in der Welt, 6. Aufl. Berlin 1958; H. Plessner, Die Frage nach der conditio humana, Frankfurt a.M. 1976; W. Kudlich, Philosophische Anthropologie, Mannheim 1973; J. Fischer, „Philosophische Anthropologie“. Eine Denkrichtung des 20. Jahrhunderts, Freiburg 2008; vgl. auch C. Bumke, Menschenbilder des Rechts, JöR n.F. 57, Tübingen 2009, S. 125 ff.

57 Keine Anthropologie im hiesigen Sinn ist die kantische; I. Kant fokussiert sich in seinem Werk „Anthropologie in pragmatischer Hinsicht“ (1. Aufl. 1798) nicht auf den kreatürlichen Mensch, sondern auf den Menschen als sittliche, also bereits normativ beschriebene Person.

58 Für den Bereich der Rechtssoziologie zu nennen ist H. Schelsky; vgl. dazu mit den entsprechenden Nachweisen Raiser, Rechtssoziologie (Fn. 10), S. 148 (154). 
„Rechtsanthropologie “59 oder „anthropologische Rechtsphilosophie“ hat sich nicht herausgebildet ${ }^{60}$ - obwohl bereits M.T. Cicero das Forschungsprogramm einer Rechtsanthropologie vorformuliert hat: „Natura iuris explicanda nobis est eaque $\mathrm{ab}$ hominis repetenda natura “. ${ }^{61}$ Soweit rechtsanthropologische Forschungsansätze bestehen, sind zwei grundlegend verschiedene methodische Herangehensweisen zu unterscheiden:

- Auf der einen Seite steht ein empirisches Verständnis, dessen Anliegen in erster Linie das Beobachten und Beschreiben des tatsächlichen Funktionierens von Recht in verschiedenen, auch ursprünglichen Gesellschaftsformen ist. ${ }^{62}$ Rechtsanthropologie ist in diesem Verständnis weniger eine normative als eine empirisch-analytische Wissenschaft.

- Die rechtsphilosophisch orientierte „Variante“ der Rechtsanthropologie zielt darauf ab, aus der condicio humana Rechtsinhalte materiell zu legitimieren. Solche Ansätze sind in der deutschsprachigen Rechtsphilosophie vereinzelt geblieben: $\mathrm{Zu}$ nennen sind die Werke von E.-J. Lampe, ${ }^{63} \mathrm{~J}$. van der Ven ${ }^{64}$ und jüngst auch von W. Brugger. ${ }^{65}$ Einen im Grundansatz anthropologischen Weg der Rechfertigung von Rechtsinhalten geht auch A. Kaufmann mit seiner personalen Verhältnislehre. ${ }^{66}$ Und auch N. Hoerster wählt für die (auch nach seiner Meinung mögliche intersubjektive) Rechtfertigung bestimmter Rechtsinhalte einen durchaus ähnlichen Weg, wenn er auf das „aufgeklärte“, „wohlverstandene“ (also natürliche?) Interesse des Menschen abstellt. ${ }^{67}$ Aus der angloamerikanischen Philosophie ist M. Nussbaum zu nennen, die in ihrem sog. „capabilities approach“ den norma-

59 Vgl. aber E.-J. Lampe, Rechtsanthropologie. Entwicklungen und Probleme, ARSP 85 (1999), S. 246 sowie das Beiheft 22 (1985) zum ARSP mit dem Titel „Beiträge zur Rechtsanthropologie“; R. Lange, Anthropologie und Recht, FS 600 Jahre Universität zu Köln, Köln 1988, S. 459 ff.

60 W. Fikentscher, Methoden des Rechts in vergleichender Darstellung, Bd. 1, Tübingen 1975, S. 60.

61 M.T. Cicero, De legibus, I, 17; deutsch: „Das Wesen des Rechts ist nämlich zu erklären, und dieses ist vom Wesen des Menschen abzuleiten."

62 Ein Hauptrepräsentant dieser Richtung ist L. Pospisil, Anthropologie des Rechts; Recht und Gesellschaft in archaischen und modernen Kulturen, München 1982; dazu P. Landau, Anthropologie des Rechts, FamRZ 1986, S. 126; ferner: W. Fikentscher, Rechtsanthropologie - Am Beispiel einer Feldstudie zu rechtlichen Reaktionen auf Gewalt in der Familie bei Pueblo-Indianern, Jura 1998, S. 182 (insbes. zur sog. „Denkstilforschung“).

63 E.-J. Lampe, Rechtsanthropologie. Eine Strukturanalyse des Menschen im Recht, 1. Band, Berlin 1970, S. 7: „,dass das Recht strukturell an den Einzelmenschen gebunden ist und gebunden sein muss, sofern es den Anspruch erhebt, richtiges Recht zu sein. "; ders., Grenzen des Rechtspositivismus. Eine rechtsanthropologische Untersuchung, Berlin 1988; ders., Anthropologische Legitimation des Grundgesetzes, in: W. Brugger (Hrsg.), Legitimation des Grundgesetzes aus Sicht von Rechtsphilosophie und Gesellschaftstheorie, Baden-Baden 1994, S. 189.

64 J.J.M. van der Ven, Ius humanum. Das Menschliche und das Rechtliche, Frankfurt a.M. 1981.

65 W. Brugger, Würde, Recht und Rechtsphilosophie im anthropologischen Kreuz der Entscheidung, in: W. Brugger/U. Neumann/S. Kirste (Fn. 7), S. 50; ders., Das anthropologische Kreuz der Entscheidung in Politik und Recht, 2. Aufl. Baden-Baden 2008; H. Joas/M. Jung (Hrsg.), Über das anthropologische Kreuz der Entscheidung, Baden-Baden 2008.

66 Kaufmann, Rechtsphilosophie (Fn. 30) S. 292 ff: Die „Person als Grundrelation des Rechts“.

67 N. Hoerster, Ethik und Interesse, Stuttgart 2003, S. 162; ders., Was ist Moral?, München 2008, S. 55. 
tiven Schutz moralisch schützenswerter Fähigkeiten des Menschen in den Mittelpunkt einer gerechtigkeitsorientierten Sozial- und Rechtsphilosophie stellt. Menschliche Fähigkeiten - worunter Nussbaum auch menschliche Bedürfnisse fasst - sind Bedingungen eines gelungenen (durchaus im aristotelischen Sinne) Lebens und daher moralischer (und auch rechtlicher) Schutz- und Fördergegenstand, über die nationalstaatlichen Grenzen hinaus. ${ }^{68}$

\section{F. Das Argumentationsprogramm einer anthropologischen Rechtsphilosophie \\ I. „Naturrechtsverdacht“}

Das Legitimationsmodell einer anthropologischen Rechtsphilosophie steht methodisch unter Naturrechts-„,Verdacht“. Zwei Einwände sind zu erwarten:

- Ein Abstellen auf die condicio humana sei zum Scheitern verurteilt, da diese nicht erkennbar, seit jeher umstritten sei, so dass es sich doch um eine substanzontologische Setzung handele. Dem ist entgegenzuhalten, dass es dem hier vertretenen Legitimationsprogramm nicht um Letztbegründung (eine solche wäre in der Tat nicht möglich), sondern lediglich um die Plausibilität normativer Ableitungen geht.

- Der zweite Einwand ist der Vorwurf des "Sein-Sollen-Fehlschlusses“ - man dürfe nicht aus der tatsächlichen condicio humana normative Schlüsse ableiten. Auch dieser Einwand lässt sich entkräften. ${ }^{69}$ Der unzulässige Fehlschluss betrifft bzw. verbietet nur zwei Ableitungsmodalitäten: Erstens folgt aus einem tatsächlichen Zustand nicht, dass dieser normativ richtig oder falsch ist. Zweitens lässt sich nicht im Sinne einer Letztbegründung dartun, dass aus einem bestimmten tatsächlichen Zustand bestimmte normative Konsequenzen zwingend zu ziehen sind. Kein „Fehlschluss“ liegt indes in der Behauptung, ein bestimmter Zustand könne ein plausibler Grund sein, daran normativ Folgen zu knüpfen - nur darum geht es hier.

\section{Methodische Anforderungen an die Annahme von Plausibilität}

Auch die Behauptung von Plausibilität bedarf indes hinreichender Begründung: unter welchen Bedingungen ist eine normative Ableitung aus einem tatsächlichen Phänomen plausibel? Auch wenn es keine Letztbegründung geben kann, muss das Begründungsniveau doch qualifiziert sein, um überzeugen zu können. Zwei Begründungsstrategien seien hier vorgeschlagen: ${ }^{70}$ die sog. „Konvergenztheorie“ der Wahrheit (sogleich 1.) sowie die Kategorie des transzendentalen Interesses (unten 2.).

68 Vgl. etwa M. Nussbaum, Frontiers of Justice. Disability, Nationality, Species Memebrship, Cambridge 2006.

69 Zum Sein-Sollen-Problem s. eingehend G. Ellscheid (Fn. 45), S. 201.

70 Anknüpfend an J.F. Lindner, Zur Kategorie des rechtswertungsfreien Raumes aus rechtsphilosophischer Sicht, ZRPh 2004, S. 87. 


\section{Die Konvergenztheorie der Wahrheit}

Die Konvergenztheorie der Wahrheit ist - wie die Korrespondenz- und die Konsensustheorie - den philosophischen Wahrheitstheorien zuzuordnen: ${ }^{71}$ Wesentliches Kriterium für die Richtigkeit einer normativen Aussage ist nach ihr nicht allein ein verfahrensmäßig (etwa im idealen Diskurs) hergestellter Konsens, sondern der Umstand, dass mehrere von einander unabhängige Subjekte hinsichtlich desselben Gegenstandes verfabrensunabhängig zu inhaltlich übereinstimmenden Thesen gelangen. Die verfahrensunabhängige Konvergenz von Aussagen ist ein gewichtiges Indiz für ihre Richtigkeit. Normative Aussagen wie „gleiche Würde“, „gleicher Wert“ aller Menschen, „gleiches Lebensrecht für alle Menschen“, Freiheit und Selbstbestimmung, Solidarität und Chancengleichheit und weitere Menschenrechte weisen ein hohes Maß an Konvergenz auf. Sie werden in vielen (wenn auch nicht allen) Ländern, in vielen Verfassungstexten und in der internationalen Gemeinschaft als gerecht empfunden und normativ anerkannt. Da konvergent begründete außerrechtliche Wertungen ein qualifiziertes Begründungsniveau aufweisen, ist es plausibel, dass sie vom Recht übernommen werden.

\section{Die Kategorie des transzendentalen Interesses}

Die zweite (stärkere) Legitimationsstrategie für die Begründung materieller Plausibilität ist der Bezug auf die Kategorie des transzendentalen Interesses. ${ }^{72}$ Diese analytische Argumentationsfigur thematisiert die Bedingung für die Möglichkeit menschlicher Interessen überhaupt. Ein transzendentales Interesse ist ein solches, das logischer Weise verwirklicht sein muss, damit alle oder doch eine Vielzahl anderer menschlicher Interessen realisiert werden können. Das höchste transzendentale Interesse ist das Lebensinteresse: Nur wer lebt, kann überhaupt irgendein anderes Interesse realisieren. Leben ist Bedingung für die Möglichkeit von Interessenrealisierung und damit das analytisch höchstrangige Interesse. Ein Legitimationssatz, der den gleichen Wert und die gleiche Schutzwürdigkeit transzendentaler Interessen behauptet, ist damit zwar nicht als „richtig“ bewiesen ${ }^{73}$ er weist jedoch ein analytisch

71 A. Kaufmann, Rechtsphilosophie (Fn. 30) S. 283.; L.B. Puntel, Wahrheitstheorien in der neueren Philosophie, 2. Aufl. Darmstadt 1993; K. Gloy, Wahrheitstheorien, Tübingen - Basel 2004.

72 Vgl. zu diesem Begriff M. Kettner, Höffes transzendental-kontraktualistische Begründung der Menschenrechte, in: W. Kersting (Hrsg.), Gerechtigkeit als Tausch? Frankfurt a.M. 1997, S. 243 (254); O. Höffe, Demokratie im Zeitalter der Globalisierung, München 1999, S. 55. Prägnant Kersting, Politische Philosophie (Fn. 52), S. 47: „Die Interessen der ersten Klasse sind fundamentale Interessen, Interessen erster Ordnung; man kann sie auch als transzendentale Interessen bezeichnen, weil ihre zufriedenstellende Berücksichtigung die Bedingung der Möglichkeit normalen Lebens und alltäglichen interessenverfolgenden Handelns überhaupt ist. Ihr Inhalt ist die Sicherung der menschlichen Grundgüter, des Lebens, der körperlichen Unversehrtheit, der Freiheit. Die Interessen der zweiten Klasse sind kontingente Interessen, Interessen zweiter Ordnung; sie können sich erst dann ausbilden und handlungswirksam entfalten, wenn die Interessen erster Ordnung befriedigt sind. "

73 In einer solchen Beweis-Annahme läge ein unzulässiger Sein-Sollen-Fehlschluss. 
hohes Begründungsniveau auf, das es plausibel erscheinen lässt, daran auch rechtlich anzuknüpfen. ${ }^{74}$

\section{Die „condicio humana“ als Erkenntnisquelle gerechten Rechts}

Ein für eine materielle Plausibilitätsthese hinreichend qualifiziertes Begründungsniveau weisen mithin Aussagen auf, die konvergente Zustimmung finden (können) und/oder den Schutz und die Realisierung transzendentaler Interessen betreffen. Es ist plausibel, dass nach diesen Maßstäben begründbare Legitimationsmaßstäbe von der Rechtsordnung (und damit auch von der Rechtswissenschaft) aufgegriffen werden. Vier Dimensionen der condicio humana dürften sich dementsprechend als materielle Quelle plausibler Normbegründung erweisen lassen: das Existenzielle (1.), das Substanzielle (2.), das Soziale (3.) sowie das Innerliche (4.).

\section{Das Existenzielle}

Existenz ist die transzendentale Grundlage jedes Menschseins. Nur durch die körperlich-biologische Existenz ist Menschsein möglich - zumindest nach derzeitigem Stand der Wissenschaft. Daran knüpfen sich drei normative Ableitungszusammenhänge.

- Die gleiche Existenz als Mensch: Jeder Mensch gehört biologisch der Gattung „Mensch“ an und dies in gleicher Weise. Gleichheit im Sein ist nicht hinwegdefinierbarer Teil der condicio humana. Eine unterschiedliche normative Behandlung der Menschen ist damit zwar nicht ausgeschlossen, aber nicht apriorisch feststehend oder begründbar, vielmehr aposteriorisch normativ rechtfertigungsbedürftig. Gleichheit ist mithin ein transzendentales Interesse: Apriorisch begründete Gleichheit in der condicio humana ist Bedingung für die Möglichkeit normativer Gleichheit, gleicher Würde im Sinne der Gleichwertigkeit jedes soseienden Menschen. ${ }^{75}$ Jede Ungleichbehandlung bedarf der normativen Legitimation.

- Der Mensch ist als „Mängelwesen“ (Gehlen) in seiner Existenz bedroht: durch andere Menschen, durch Tiere, Naturgewalten, Krankheiten, durch sich selbst. Der Bedrohung entspringt das Bedürfnis nach Existenz-Gewissheit, nach Schutz („Überlebenstrieb“). Da der Mensch Schutz nicht allein, jedenfalls nicht in erforderlichem Umfang herstellen kann, bedarf er der Gemeinschaft mit anderen Menschen. Nur in der Gemeinschaft ist dauerhafter Schutz möglich. Da aber die (noch nicht normativ gedachte!) Gemeinschaft ihrerseits Quelle von Bedrohung und

74 In der Rechtsprechung des BVerfG klingt die Figur des transzendentalen Interesses an: „Das menschliche Leben stellt innerhalb der grundgesetzlichen Ordnung einen Höchstwert dar; es ist die vitale Basis der Menschenwürde und die Voraussetzung aller anderen Grundrechte" (BVerfGE 39, 1, (42)). Aus Sicht der Rechtsanthropologie s. E.-J. Lampe, Anthropologische Legitimation des Grundgesetzes, in: W. Brugger (Fn. 63) S. 189 (198) „,biotische Legitimationsgründe“. Zur Bedeutung der Kategorie des transzendentalen Interesses für die Grundrechtsdogmatik s. J.F. Lindner, Theorie der Grundrechtsdogmatik, Tübingen 2005, S. 205 (dort Fn. 114).

75 Lindner, Grundrechtsdogmatik (Fn. 74), S. 180 (für das Menschenwürdekonzept des Grundgesetzes). 
Zwang ist, bedarf auch diese selbst der Zügelung durch Regeln, denen sich die Menschen unterwerfen. ${ }^{76}$ Normative Regeln und Sanktionen sowie Institutionen zur Durchsetzung solcher Regeln zur Verfügung zu stellen ist Aufgabe des Rechts, dieses zu gewährleisten Aufgabe des Staates (oder vergleichbarer, das Gewaltmonopol beanspruchender Institutionen).

- Damit untrennbar ist eine aposteriorisch-institutionelle Bedrohtheit der menschlichen Existenz verbunden. Staat, Gemeinden oder sonst normativ geschaffene oder anerkannte Institutionen (öffentlicher oder privater Natur) sind dem einzelnen Menschen nicht nur Schutz und Gewissheit, sondern auch Gefahr. Der in seiner Existenz bedrohte Mensch bedarf auch des Schutzes vor den zu seinem Schutz existierenden rechtlichen Institutionen. Das ist die ehrwürdige Aufgabe der Grundrechte: Schutz vor Willkür und Unverhältnismäßigkeit öffentlich-institutioneller (zumal staatlicher) Macht und zugleich Schutz vor privat-institutioneller (zumal unternehmerischer) Macht durch die öffentlich-institutionelle Macht. Kurz: Schutz des Menschen vor staatlicher Macht und Schutz vor privater durch staatliche Macht. ${ }^{77}$ In dieser - allerdings nur auf den ersten Blick - paradoxen Struktur findet die gesamte Rechtsordnung ihren Kristallisationspunkt (zur Figur des Interessendreiecks s. unten G.III.2.).

\section{Das Substanzielle}

Der Mensch existiert nicht nur, er ist nicht nur bedroht und auf Schutz verwiesen, er ist vielmehr mit Verstand und Vernunft ausgestattet. Diese bilden das Substanzielle des Menschseins, konstituieren überhaupt die Möglichkeit, frei zu sein, frei nicht nur instinktiv - zu handeln, sich selbst zu bestimmen, Kultur hervorzubringen, Humanität zu zeigen und Emotionalität zu erzeugen. Dieses Substanzielle (im Sinne eines transzendentalen Interesses) kommt jedem Menschen zu - auch dann wenn er davon aktuell (etwa wegen Ohnmacht, Koma, Behinderung) keinen Gebrauch machen kann. Die (gleichberechtigte) Entfaltung dieses Substantiellen zu ermöglichen und vor Behinderungen zu schützen ist plausibler Anknüpfungspunkt für eine gerechte Rechtsordnung. Dieser Entfaltung dienen die Freiheitsrechte und eine diese effektiv realisierende Rechtsordnung, insbesondere Privatrechtsordnung.

\section{Das Soziale}

Hinzukommt die distributiv-soziale Dimension des Menschseins, die darauf verweist, dass der Mensch zur Verwirklichung seiner Interessen Mittel und tatsächliche Chancen benötigt, die er sich selbst nicht oder nur teilweise beschaffen kann. Damit die ideell-kulturelle Dimension des Menschseins (das „Substanzielle“) mit Leben erfüllt werden kann, benötigt der Mensch materielle Mittel sowie das, was man heute

76 Grundsätzlich zu diesen Legitimationszusammenhängen Höffe, Politische Gerechtigkeit (Fn. 36).

77 Die plastische Formel lautet: Schutz durch Eingriff; R. Wabl/J. Masing, Schutz durch Eingriff, JZ 1990, S. 557. 
unter dem Schlagwort „Bildung“ versteht. Bildung ist ein transzendentales Gut, da durch sie die Realisierung vieler anderer Interessen erst ermöglich wird. Wichtiges Thema einer gerechten Rechtsordnung ist die Beachtung von Verteilungsgerechtigkeit in ideeller und materieller Hinsicht. Die ideelle Komponente wird rechtlich abgebildet durch den Grundsatz der Chancengerechtigkeit. Diese meint den gleichen, vorurteils- und bedingungslosen Zugang jedes Menschen zu den hoheitlichen Verteilungsprozessen, in denen es nicht um die Zuteilung von materiellen Gütern, sondern von Chancen zur Selbstentfaltung geht. Überall dort, wo die freie persönliche, berufliche etc. Entfaltung des Einzelnen von hoheitlich postulierten Voraussetzungen abhängt, muss jedem Einzelnen die gleiche rechtliche Chance eingeräumt werden, diese Voraussetzungen zu erfüllen. Die materielle Komponente der Verteilungsgerechtigkeit wird zumal durch das Sozialstaatsprinzip und das dieses konkretisierende Arbeits- und Sozialrecht abgebildet. Eine „richtige“ Rechtsordnung muss mithin auch geprägt sein durch ein chancengerechtes Bildungs ${ }^{78}$ - und Berufszugangsrecht sowie durch ein materiell gerechtes Güterverteilungsrecht. ${ }^{79}$ Insoweit gibt es indes einen breiten (demokratischen) Einschätzungsspielraum. Materiell gerecht ist nicht nur eine bestimmte Verteilungsform, es sind ganz unterschiedliche Modelle denkbar, die als gerecht bezeichnet werden können.

\section{Das Innerliche}

Der Mensch existiert nicht nur als „animal rationale“, er ist auch ein (nicht hinwegdefinierbar) innerliches Wesen: psychisch nicht stets stabil, mitunter zweifelnd, sich sorgend, nach Gewissheit strebend. Er verhält sich nicht stets streng rational, ist kein „homo oeconomicus“, sondern in seinen Entscheidungen bisweilen wankelmütig, unberechenbar, von Launen, Vorurteilen, Tagesstimmungen, irrationalen Präferenzen, Freuden und Ängsten geleitet. Überschwang, Euphorie, Leichtsinn, Neid ${ }^{80} \mathrm{Wi}$ dersprüchlichkeit gehören unauflöslich zur condicio humana. Eine Rechtsordnung, die gerecht sein will, hat dieses Innerliche zu berücksichtigen, zumindest in Rechnung zu stellen, auch wenn das „forum internum“ häufig unzugänglich und im Dunkeln bleiben wird. Es muss in Abwägungsprozessen, z.B. im Rahmen der Strafzumessung

78 J.F. Lindner, Was ist und wozu brauchen wir eine Theorie des Bildungsrechts? DÖV 2009, S. 306.

79 Zum Stand der Diskussion zwischen eher egalitären/egalitaristischen und eher libertären Verteilungsmodellen s. S. Gosepath, Gleiche Gerechtigkeit. Grundlagen eines liberalen Egalitarismus, Frankfurt a.M. 2004, insbes. S. 215; W. Hinsch, Gerechtfertigte Ungleichheiten. Grundsätze sozialer Gerechtigkeit, Berlin - New York 2002; W. Kersting, Theorien der sozialen Gerechtigkeit, Stuttgart 2000; A. Krebs (Hrsg.), Gerechtigkeit oder Gleichheit. Texte der neuen Egalitarismuskritik, Frankfurt a.M. 2000; Übersicht bei J.F. Lindner, ARSP 91 (2005), S. 446. Eine Grenze finden Verteilungsmodelle allerdings ihrerseits an den Freiheitsrechten, wenn sie mit Umverteilungen verbunden sind, die den Raum selbstbestimmter Entfaltung übermäßig einschränken, etwa durch übermäßig hohe Steuern. Daran müssen sich auch jüngst (wieder) diskutierte Modelle eines bedingungslosen Grundeinkommens messen lassen, vgl. dazu die Diskussion im Roman Herzog Institut, Bedingungsloses Grundeinkommen - eine Perspektive für die soziale Marktwirtschaft? München 2008.

80 Vgl. dazu M. Desens, Neid als Grundrechtsausübungsmotiv, AöR 133 (2008), S. 404. 
oder bei der Gestaltung des Prüfungsrechts eine Rolle spielen (dürfen); zu weiteren Beispielen s.u. G.III.1.

\section{G. Mögliche Konsequenzen für die Rechtswissenschaft}

Was kann aus dem soeben in freilich nur groben Zügen gezeichneten Legitimationsprogramm einer anthropologischen Rechtsphilosophie für die Rechtswissenschaft insgesamt folgen? Hat das Recht die Aufgabe, aus der condicio humana ableitbare Gerechtigkeitsinhalte zu gewährleisten, muss die Rechtswissenschaft im Grundsatz darauf bezogene Gerechtigkeitswissenschaft sein. Der Dienst an der Gerechtigkeit ist Paradigma der Rechtswissenschaft, darauf sind alle noch so verzweigten Einzelrechtsgebiete zurückzuführen, daran sind alle noch so technischen und komplexen dogmatischen Strukturen und Konstruktionen legitimatorisch zu spiegeln. An diesem Maßstab orientiert könnten sich einzelne Bereiche, Teilgebiete der Rechtswissenschaft wie folgt systematisieren lassen. ${ }^{81}$

\section{Rechtsphilosophie: Die Begründung gerechter Rechtsinhalte}

Forschungsgegenstand der (materiell verstandenen) Rechtsphilosophie ist die Begründung der Inhalte einer gerechten Rechtsordnung. Die Begründung der Plausibilität von Richtigkeitsmaßstäben unter Bezugnahme auf die condicio humana ist gewissermaßen „Kerngeschäft“ der Rechtsphilosophie. Diese Aufgabe ist durch die abstrakte Benennung der Quellen gerechten Rechts (oben F.III.) natürlich noch keineswegs erfüllt. Die materiellen Ableitungen aus der condicio humana müssen vielmehr fortwährend konkretisierend präzisiert und im Hinblick auf die momentanen und künftigen (gesellschaftlichen, bioethischen, global-humanen, global-klimatischen, global-sicherheitlichen etc.) Herausforderungen an die (deutsche, europäische und internationale) Rechtsordnung aktualisiert werden. ${ }^{82}$

\section{Rechtspolitik: Die Umsetzung gerechter Rechtsinhalte}

Mit dem Postulat bestimmter Rechtsinhalte als richtig ist es nicht getan. Die materiellen Inhalte müssen (auf allen Rechtsetzungsebenen) in geltendes (nationales, europäisches und internationales) Recht umgesetzt werden. Diese Realisierungsaufgabe wird heute als Aufgabe der Politik verstanden und damit dem Spiel der politischen Kräfte, in unseren Breitengraden dem demokratisch organisierten, primär parteipo-

81 Gegenstand der nachfolgend nicht eigens behandelten Rechtsgeschichte wäre die Analyse der anthropologischen Gerechtigkeitsmaßstäbe auf der Schiene der Zeit und die Aufbereitung der jeweils gefundenen ge- und misslungenen Regelungsmodelle. Rechtssoziologie, Rechtspsychologie und Rechtsökonomie dienen maßgeblich der empirischen Vergewisserung des Für-Richtig-Gehaltenen und der daraus entwickelten Rechtsregeln.

82 Beispiel: Die selbstbestimmte Entfaltung des Menschen gehört zum Substanziellen der condicio humana. Daraus lassen sich Elemente einer freiheitlichen Rechtsordnung postulieren. Die These der jüngeren Hirnforschung, es seien Zweifel an der Willensfreiheit des Menschen anzumelden, stellt der Rechtsphilosophie die Aufgabe, was daraus für die Legitimation einer Freiheitsrechtsordnung folgt; s. dazu auch unten IV.2.(3). 
litischen Meinungsbildungsprozess überantwortet. Gleichwohl sollte Rechtspolitik auch als Aufgabe der Rechtswissenschaft begriffen werden: Politik bedeutet im „modernen“ Staat Gestaltung maßgeblich durch Recht. Diese Gestaltung sollte nicht allein Interessenverbänden und der auf Wiederwahl verwiesenen Parteipolitik überlassen werden. Es ist durchaus eine gewichtige Aufgabe der Rechtswissenschaft, die plausibel begründbaren Gerechtigkeitspostulate auch politisch einzufordern. Rechtswissenschaft sollte insofern auch normativ-politische Wissenschaft sein. Der Verweis auf den politischen Gestaltungsspielraum des demokratisch legitimierten Verfassungs- oder Gesetzgebers darf kein Feigenblatt der Rechtswissenschaft dafür sein, unabdingbare Gerechtigkeitspostulate nicht geltend zu machen. Freilich wird es in vielen Problembereichen mehrere Lösungsansätze geben, die den plausiblen Richtigkeitsmaßstäben gerecht werden und daher dem demokratischen Findungsprozess überantwortet werden können - insofern ist es dann Aufgabe der Rechtswissenschaft, vorhandene Spielräume für demokratische Dezisionen aufzuzeigen. Insgesamt sollte die Rechtswissenschaft, will sie sich als normativ arbeitende Wissenschaft behaupten, das Feld der Rechtspolitik nicht gänzlich der ökonomischen und politikwissenschaftlichen Beratung oder gar der Steuerung durch sog. „Think-Tanks“ überlassen.

\section{Rechtsdogmatik: Entwicklung einer gerechtigkeitsoptimierenden Anwendungslehre}

\section{Aufgabe der Dogmatik}

Zur materiellen Begründung von Richtigkeitsmaßstäben durch die Rechtsphilosophie und deren Umsetzung durch die Rechtspolitik hinzukommen muss als dritte Aufgabe der Rechtswissenschaft die dogmatische Entfaltung und Ausdifferenzierung des für legitim erkannten (und eingeforderten) positiven Rechts. Die Existenz von Rechtssätzen, die den Legitimationsanforderungen gerecht werden (wie z.B. im Bereich des Öffentlichen Rechts die Freiheits- und Gleichheitsrechte, das Sozialstaatsprinzip, die grundrechtlichen Schutzpflichten etc.), genügt für sich alleine nicht. Die Funktionsfähigkeit einer gerechten Rechtsordnung setzt deren Anwendung voraus. Die Anwendung impliziert die Entwicklung einer Dogmatik, die eine sach- und funktionsgerechte sowie berechenbare Handhabung des Rechts durch die beteiligten Akteure im Einzelfall ermöglicht. Dies ist Aufgabe der Rechtsdogmatik, die dabei den steten Bezug zu den materiellen Legitimationsgrundlagen erkennbar machen und diese im Einzelfall praktisch werden lassen muss. Die Rechtsdogmatik erfüllt diese Aufgabe durch die Formulierung von abstrakten Begrifflichkeiten, Argumentationsfiguren, Prüfungsschemata und bereichsübergreifenden Strukturmodellen zur praktischen Anwendung des Rechts. Am Beispiel des Öffentlichen Rechts: Das Grundgesetz hat eine Rechtsordnung errichtet, die nach dem hier vertretenen Legitimationskonzept „gerecht“ ist: Gleicher Schutz der Würde für alle Menschen, Freiheits- 
entfaltung durch Entfaltungsgrundrechte, Integritätsschutz durch Integritätsgrundrechte (und Schutzpflichten) sowie (ideelle und materielle) Verteilungsgerechtigkeit durch den Gleichheitssatz und das Sozialstaatsprinzip. Damit sind allerdings nur die verfassungsrechtlichen Rechtsinstitute benannt. Um diesen zu effektiver Entfaltung zu verhelfen und mit Leben zu erfüllen, bedarf es der Entwicklung dogmatischer Strukturen. Solche dogmatischen Strukturen zu entwickeln ist eine der wesentlichen Aufgaben der Rechtswissenschaft, im Einzelnen der Wissenschaft vom Öffentlichen Recht. Die selbstbestimmte Freiheitsentfaltung, das Substanzielle des Menschen (oben F.III.2.) wird dogmatisch realisiert und optimiert durch das in der Grundrechtsdogmatik weitgehend anerkannte Regel-Ausnahme-Verhältnis zugunsten der Freiheit des Einzelnen: Regel ist die lückenlose Ausgangsvermutung zugunsten der Freiheit Jedermanns, die Einschränkung die rechtfertigungsbedürftige Ausnahme. Im Rahmen der Rechtfertigung kommt dann die Schutzbedürftigkeit, das Existenzielle des von der Freiheitsausübung betroffenen Dritten (oben F.III.1.) zum Tragen. Die Schutzpflicht des Staates zu Gunsten des Dritten kann den Eingriff in die Freiheitsentfaltung des Anderen rechtfertigen. Das Regel-Ausnahme-Verhältnis zugunsten der Freiheit und der Schutz durch Eingriff gehören zum dogmatischen Kernbestand einer gerechtigkeitsorientierten Rechtsordnung. ${ }^{83}$ Es setzt sich im Allgemeinen und weiten Teilen des Besonderen Verwaltungsrechts fort. Die materielle und ideelle Verteilungsgerechtigkeit, das Soziale (oben F.III.3.), wird dogmatisch ausgeformt durch die dogmatische Konkretisierung des Sozialstaatsprinzips und in einem am Maßstab der Chancengleichheit ausgearbeiteten Rechtsgebiet „Bildungsrecht“ .84

Wichtige Ausprägung des hier vertretenen rechtsanthropologischen Ansatzes ist es, bei der dogmatischen Ausgestaltung (und Anwendung) des Rechts auch der (zumindest) typisierbaren Befindlichkeit des Menschen Rechnung zu tragen, sprich: die innere Dimension der condicio humana zu beachten. Die Rechtsdogmatik könnte und sollte in stärkerem Maße als die verfahrensorientiert-funktionalen Steuerungsansätze dies tun eine anthropologische Perspektive einnehmen. Dazu drei Beispiele (überwiegend aus dem Bereich des Öffentlichen Rechts):

- Im Bildungsrecht, das trotz seiner fundamentalen Bedeutung für jeden einzelnen Menschen in der Rechtswissenschaft bislang keine nennenswerte Rolle spielt, geht es um Regelungen, die ganz wesentlich über menschliche Weggabelungen entscheiden: über den Zugang zu weiterführenden Schulen und damit über Bildungschancen, über den Zugang zu Hochschulen und damit über Berufschancen. Eine essentielle Bedeutung in diesem Rahmen hat das Prüfungsrecht, das die spezifi-

83 Vgl. dazu J.F. Lindner, Das Regel-Ausnahme-Verhältnis im Verwaltungsrecht, VerwArch 98 (2007), S. 213.

84 Die dogmatische Durchdringung eines Bildungsrechts im weiteren Sinne befindet sich noch in der Anfangsphase, vgl. dazu Lindner, Theorie des Bildungsrechts (Fn. 78). 
schen Befindlichkeiten des Prüflings berücksichtigen und ihm regelmäßig eine „zweite Chance“ einräumen muss. ${ }^{85}$

- Das zweite Beispiel betrifft das Medien- und Strafverfahrensrecht: Vom anthropologischen Ansatz her geboten erscheint eine stärkere Berücksichtigung des allgemeinen Persönlichkeitsrechts in der öffentlichen Berichterstattung insbesondere im Hinblick auf Beschuldigte. Der öffentlichen Berichterstattung über Strafverfahren unter Namensnennung wird heute in einer überwiegend funktional-technokratischen Sichtweise zu Gunsten einer Theorie des öffentlichen Diskurses (in der Praxis handelt es sich zumeist um die Befriedigung von Sensationslust und damit zu erreichende Auflagensteigerung) zu häufig ein Vorrang gegenüber dem allgemeinen Persönlichkeitsrecht des Beschuldigten sowie der Unschuldsvermutung eingeräumt. Ist der einzelne Beschuldigte durch die namentliche Berichterstattung auch für sein Leben lang ruiniert, so sei dies doch durch die Bedeutung der Medien für die Herstellung von Öffentlichkeit als Bedingung für das Funktionieren von Demokratie gerechtfertigt. Ein von einem rechtsanthropologischen Standpunkt aus gesehen schwer erträgliches Ergebnis! ${ }^{86}$ Generell muss ein anthropologischer Ansatz stärker auf den Schutz des Einzelnen vor öffentlicher Funktionalisierung abzielen. Unterstützung erhält dieses Ansinnen jüngst durch den Europäischen Gerichtshof für Menschenrechte (Stichwort: „Caroline“-Rechtsprechung).

- Das dritte Beispiel sei dem Verwaltungsprozessrecht entnommen: Dessen Paradigma ist, dass klageberechtigt nur ist, wer geltend machen kann, in eigenen Rechten verletzt zu sein ( $\$ \$ \$ 42$ II, 47 II VwGO). Ganze Bibliotheken befassen sich seitdem mit der Frage, wann eine Vorschrift des Öffentlichen Rechts „drittschützende" Wirkung hat. Studierende verbringen Unmengen an (wertvoller) Zeit mit dieser aus hiesiger Sicht eher entbehrlichen Frage. Aus der Sicht eines anthropologischen Ansatzes greift das Rechtsschutzparadigma zu kurz, kann doch der Einzelne auf Grund tatsächlicher (auch innerer) Umstände ein ganz spezifisches Interesse an der Überprüfung einer (nicht an ihn selbst gerichteten) Verwaltungsentscheidung haben, selbst wenn er sich nicht auf eine drittschützende Norm berufen kann. Es wäre also zu überlegen, für $\mathbb{S} \$ 42$ II, 47 II VwGO ein berechtigtes Interesse an der gerichtlichen Überprüfung ausreichen zu lassen. Hiergegen wird eingewandt werden, das käme einer dem deutschen Recht unbekannten Popularklage gleich. Ist das ein Gegenargument? Der Freistaat Bayern kennt seit langem

85 Vgl. dazu z.B. J.F. Lindner, Wider unnötige Härten im Prüfungsrecht“, RdJB 2008, S. 218.

86 Die Bemühungen insbesondere von Seiten der Strafverteidiger um eine stärkere Berücksichtigung des Persönlichkeitsrechts von Beschuldigten sind bislang nicht wirklich erfolgreich gewesen. So hat sich z.B. der 30. Strafverteidigertag 2006 mit der Thematik befasst: „Brauchen wir eine justizielle Schweigepflicht? “; vgl. dazu J.F. Lindner, Der Schutz des Persönlichkeitsrechts des Beschuldigten im Strafverfahren“, StV 2008, S. 210; ders., Der Verfassungsrechtssatz von der Unschuldsvermutung, AöR 133 (2008), S. 235; aus der Praxis G. Friedrichsen, „Litigation-PR“ - Prozessführung über Medien? ZRP 2010, S. 263. 
die Popularklage gegen Rechtsnormen, ohne dass es zu Klageexzessen gekommen wäre. ${ }^{87}$

\section{Rechtsbereichsübergreifende Dogmatik: die Figur des Interessendreiecks}

Wichtig für die Kohärenz der Rechtsdogmatik ist die Formulierung bereichsübergreifender dogmatischer Strukturen, die die bislang weitgehend nebeneinander stehenden dogmatischen „Fächer“ Zivilrecht, Strafrecht und Öffentliches Recht stärker miteinander verbinden. Zwar muss jedes dieser Gebiete insbesondere in den bereichsspezifischen Besonderheiten eigene dogmatische Begrifflichkeiten und Strukturen herausbilden; eine allgemeine Rechtsdogmatik, die für alle drei Rechtsgebiete gelten würde, wäre aufgrund der spezifischen Eigenheiten nicht möglich oder gekünstelt, jedenfalls aber unterkomplex. Dies darf jedoch nicht den Blick darauf verstellen, dass es durchaus möglich sein könnte, übergreifende grundsätzliche rechtsdogmatische Konzepte zu formulieren, die die einzelnen Rechtsgebiete zumindest im Grundsätzlichen „zusammenhalten“.

Ein Baustein dafür wäre die Entwicklung eines allgemeinen rechtsdogmatischen Interessendreiecks: Rechtlich relevante Sachverhalte lassen sich auf bestimmte tatsächliche Interessen und Interessenkonflikte zurückführen, die in einem Interessendreieck darstellbar sind. Dessen drei Pole sind (schematisch dargestellt):

- P1, eine natürliche oder juristische Person, eine Einzelperson oder ein Unternehmen, die ein bestimmtes Interesse realisieren will,

- P2, P3, .. Pn, die durch die Interessenrealisierung des P1 in ihren Interessen beeinträchtigt wird/werden.

- Schließlich an der Spitze des Dreiecks der Staat (stellvertretend für alle Hoheitsträger, auch für die EU), der die Interessenermöglichung von P2-n und P1 im Auge haben und ein Rechtsinstrumentarium zur Bewältigung von Interessenkonflikten zur Verfügung stellt.

Dieses Rechtsinstrumentarium, das letztlich nichts anderes ist als ein Instrumentarium zur Erfüllung der Schutzpflicht zugunsten von P2-n oder anderer verfassungsrechtlich geschützter Rechtsgüter, wird dogmatisch konkretisiert in den verschiedenen Bereichen des Rechts: im Strafrecht, im Gefahrenabwehrrecht, im Arbeitsrecht, im Verbraucherschutzrecht, im Wettbewerbsrecht, im Regulierungsrecht. Stets ist das Grund-„,Schema“ das Gleiche: Der Staat ${ }^{88}$ (in Gestalt des Gesetzgebers, der Exekutive oder der Judikative) greift in rechtlich geschützte Positionen des Einen ein, um Interessenkonflikte mit einem Anderen zu einem sachgerechten Ausgleich zu brin-

88 Oder ein anderer Hoheitsträger nach Maßgabe der jeweils zugewiesenen Kompetenzen. 
gen.$^{89}$ Ein geeigneter Ansatz, die „Säulenstruktur“ der deutschen Rechtswissenschaft mindestens im Grundsätzlichen zu überwinden, könnte daher sein, dogmatisch stärker in „Lebensbereichen“ zu denken und diese Lebensbereiche dann jeweils zivil-, straf- und öffentlich-rechtlich nach Maßgabe des Interessendreiecks analytisch aufzuarbeiten.

\section{Das rechtsdogmatische Interessendreieck}

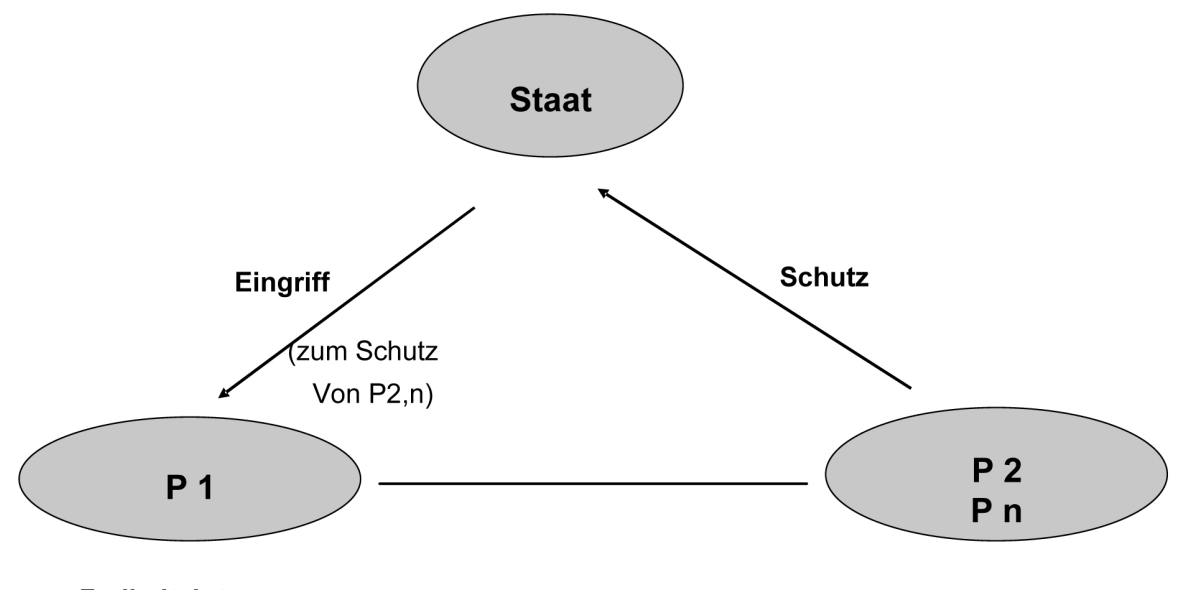

Freiheitsinteresse

Freiheitsinteresse

\section{Rechtstheorie: Rechtskritik als Aufgabe}

Ein eigenständiges rechtswissenschaftliches Fach „Rechtskritik“ existiert bislang nicht. Es könnte unter dem Dach der - bislang in ihrem Gegenstandsbereich unscharf gebliebenen - „Rechtstheorie“ eine Heimat finden. Im Gegenzug könnte mancher bislang in der Rechtstheorie verankerte Bereich, wie etwa die allgemeine Normlehre oder die Methodenlehre, eher der Rechtsdogmatik zugeordnet werden. Einer Rechtskritik als „Teilmenge“ der Rechtstheorie käme die Funktion zu, kritisch zu prüfen, ob rechtspolitische Umsetzung und dogmatische Ausformulierung den rechtsphilosophisch begründeten materiellen Maßstäben wirklich gerecht werden.

89 Die konkrete dogmatische Arbeit müsste darin liegen, für den jeweiligen Lebenssachverhalt das allgemeine Interessendreieck konkretisierend darzustellen, Interessenkonflikte zu benennen und die einschlägigen zivilrechtlichen, öffentlich-rechtlichen und strafrechtlichen Rechtsinstrumentarien zur Bewältigung der Interessenkonflikte herauszuarbeiten. 


\section{Begleitende kritische Analyse}

Dazu gehört zunächst eine begleitende Analyse der jeweiligen Vorschläge und Konzepte zur Bewältigung aktueller Herausforderungen. Aufgabe einer begleitenden Rechtskritik ist es, konzeptionelle Grundlagen für die Rechtspolitik auf der Basis des rechtsphilosophischen Programms zu formulieren. Ein besonderes Augenmerk sollte dabei auf der Optimierung der Einheit der Rechtsordnung im Sinne der Widerspruchsfreiheit liegen. ${ }^{90}$ Vermeidbare Widersprüche in der Rechtsordnung korrumpieren das Rechtsempfinden der Menschen und stellen die Rechtsordnung als Gerechtigkeitsordnung vor schwere Belastungsproben. Zwar lassen sich in einer komplexen Rechtsordnung Widersprüche und Inkonsequenzen nie völlig vermeiden. Sie bedürfen indes der kritischen Analyse. ${ }^{91}$ „Rechtskritik“ sollte insoweit Aufgabe der Rechtswissenschaft ${ }^{92}$ sein.

\section{Ideologiekritik}

Hinzu kommen sollte eine Art „Wächterfunktion“ der Rechtstheorie gegenüber Rechtspolitik und Rechtsdogmatik. Gemeint ist die Aufgabe, Einseitigkeiten und ideologische Überhöhungen, die der condicio humana nicht gerecht werden oder diese überzeichnen, zu benennen und zu kritisieren. Diese Funktion sei anhand einiger Beispiele illustriert:

- Ein gewisses „Übertreibungspotential“ wohnt dem Grundsatz der Selbstbestimmung des Menschen inne. Es liegt in der Natur des Menschen, sich selbst zu bestimmen und zu entfalten. Allerdings ist rationale Selbstbestimmung nicht alles, es sind dabei auch die Dimensionen des mitunter irrationalen Innerlichen (s.o. F.III.4.) in Rechnung zu stellen. Rationale Selbstbestimmung ist nur eine Antriebsfeder des Menschen unter mehreren, sie darf nicht überhöht, verabsolutiert werden, wie dies beispielsweise in der Diskussion um die Sterbehilfe mitunter geschieht. Eine mit dem Selbstbestimmungsparadigma begründete Zulassung der

90 Das Gebot der Widerspruchsfreiheit ist nicht nur ein konstituierendes Element jeder Theoriebildung wissenschaftlichen Anspruchs, sondern wird auch in der verfassungsrechtlichen Literatur zumeist ohne weiteres als Funktionsbedingung des Rechts vorausgesetzt; vgl. etwa H.A. Wolff, Ungeschriebenes Verfassungsrecht unter dem Grundgesetz, Tübingen 2000, S.190. P. Kirchhof, Unterschiedliche Rechtswidrigkeiten in einer einheitlichen Rechtsordnung, Heidelberg 1978, S. 8: „Die einende und friedensstiftende Kraft einer Rechtsordnung stützt sich vielmehr nur auf die Widerspruchsfreiheit der Einzelaussagen der Rechtsordnung. Die Widerspruchslosigkeit ist Geltungsgrund für eine Rechtsordnung "; H. D. Jarass, Die Widerspruchsfreiheit der Rechtsordnung als verfassungsrechtliche Vorgabe, AöR 126 (2001), S. 588. In eine ähnliche Richtung geht das Postulat der Systemgerechtigkeit der Rechtsordnung: F.-J. Peine, Systemgerechtigkeit, Baden-Baden 1985, S. 24 ff.; C. Degenhart, Systemgerechtigkeit und Selbstbindung des Gesetzgebers als Verfassungspostulat, München 1976; C. Bumke, Relative Rechtswidrigkeit, Tübingen 2004.

91 Zu den verschiedenen Modalitäten der Rechtskritik s. bereits oben Fn. 39.

92 Ähnliches gilt für die Durchsetzung des Rechts. Auch Nichtbeachtung, Nichtvollzug oder ungleicher Vollzug trotz Vollziehbarkeit können akzeptanzzerstörende Wirkung haben. Näher zu beleuchten wären in diesem Zusammenhang zudem die offenen Flanken der Durchsetzung jeder Rechtsordnung, die in Vorurteilen, Parteilichkeiten oder sonstigen Befindlichkeiten der zur Durchsetzung des Rechts, insbesondere zur Recht,,sprechung “ befugten Personen liegen; dabei handelt es sich um Aspekte der (zu stark vernachlässigten) Rechtspsychologie; vgl. auch Fn. 25 
aktiven Sterbehilfe oder der kommerziellen Suizidbegleitung (als rechtmäßig) dürfte dem Anspruch einer gerechten Rechtsordnung nicht entsprechen. ${ }^{93}$

- Ähnliches gilt für die sog. „ökonomische Theorie des Rechts“, über die in letzter Zeit intensiv diskutiert wird. ${ }^{94}$ Auch hier greift es zu kurz, allein auf die Figur des homo oeconomicus zurückzugreifen und im Rechtsverkehr stets auf ein rationales, an ökonomischem Erfolg ausgerichtetes Handeln des Einzelnen abzustellen. Berücksichtigung finden müssen vielmehr auch Erkenntnisse der Verhaltenspsychologie, die das „Innerliche“ des Menschen, seine Irrationalitäten, Vorurteile, präferenzsteuernden Emotionen etc. gebührend in Rechnung stellen. ${ }^{95}$

- Als drittes Beispiel sei die Hirnforschung genannt, in der jüngst mitunter die Auffassung vertreten wird, die Willensfreiheit des Menschen sei aus neurobiologischer Sicht eine Fiktion, weshalb man ein neues Menschenbild benötige. ${ }^{96}$ Solche Thesen müssen von Seiten der Rechtstheorie (als Rechtskritik) in ihrer Konsequenz für die Rechtsordnung nachdrücklich hinterfragt werden. Selbst wenn sie - naturwissenschaftlich - zuträfen, wäre doch die Frage zu stellen, ob sie einem normativen Menschenbild zugrunde gelegt werden müssten oder überhaupt dürften. Rechtskritik hat ganz maßgeblich die Aufgabe, den gesellschaftlich-normativen Anspruch naturwissenschaftlicher Thesen zu relativieren. In einem anthropologischen Rechtsmodell ist das menschliche, bei nahezu jedem Menschen anzutreffende Gefühl der Freiheit des Willens der überzeugendere Anknüpfungspunkt für die Rechtsordnung als die naturwissenschaftliche Analyse der Hirnfunktionen. Mit anderen Worten: Selbst wenn neurowissenschaftliche Erkenntnisse die Willensfreiheit des Menschen in Frage stellen, kann und muss normativ an ihr festgehalten werden. Sie ist (mindestens gefühlter) Teil der condicio humana.

- In der Überprüfung und Infragestellung der normativen Relevanz naturwissenschaftlicher Erkenntnisse liegt nicht wie gelegentlich behauptet ${ }^{97}$ ein Rückfall in eine mittelalterliche Rechtskultur, sondern der Versuch, kulturell und normativ gewachsene, aus rein naturwissenschaftlicher Bedingt- und Befangenheit emanzipierte und für richtig erachtete Überzeugungen (die auch intuitiv verankert sind) zu bewahren.

- Ausdruck einer Art von Ideologisierung des Rechts ist auch der heute allgemein erhobene Anspruch, das Recht müsse jeden Konflikt rechtlich entscheiden können, für jeden Konflikt eine Lösung anbieten. Ein solcher Allherrschaftsanspruch des Rechts mag zwar in den allermeisten Konfliktlagen zum Tragen kommen können, er versagt jedoch in tragischen Konflikten gleichwertiger Rechtsgüter, in

93 Zur Diskussion m.w.N. J.F. Lindner, Grundrechtsfragen aktiver Sterbehilfe, JZ 2006, S. 373.

94 Lindner, Ökonomische Theorie des Öffentlichen Rechts (Fn. 27).

95 Hierzu Eidenmüller, homo oeconomicus (Fn. 25).

96 W. Singer, Verschaltungen legen uns fest: Wir sollten aufhören, von Freiheit zu sprechen, C. Geyer (Hrsg.), Hirnforschung und Willensfreiheit, Frankfurt a.M. 2004, S. 30.

97 S. H.A. Wolff, Die Willensfreiheit und die Grundrechte, JZ 2006, S. 929. 
denen das eine Rechtsgut nur unter Aufgabe des anderen bewahrt werden kann. Paradigmatisch hierfür ist das Beispiel des Abschusses eines vollbesetzten Passagierflugzeuges, das von Entführern z.B. in ein vollbesetztes Fußballstadion zum Absturz gebracht werden soll. Für die Lösung solcher tragischer Konflikte können weder die Ethik noch das Recht eine richtige Lösung parat haben. Das Recht sollte sich der Regelung enthalten, die Rechtswissenschaft rechts(wertungs)freie Räume aushalten. ${ }^{98}$

\section{H. Ausblick: Selbstand und Interdisziplinarität}

Als (normative) Gerechtigkeitswissenschaft hat die Rechtswissenschaft eine Chance, ihren Selbstand im Konzert der Wissenschaften bewahren. Nur wenn sie sich ihrer spezifischen normativen Aufgabe bewusst ist (und bleibt) und diese auch kommuniziert, wird sie von anderen Disziplinen wahr- und ernstgenommen. ${ }^{99}$ Eine zumal terminologische Anbiederung an andere Disziplinen wirkt insoweit schwächend.Die Rechtswissenschaft muss ihren eigenen Anspruch geltend machen. Dazu formuliert sie die Methoden für die Gewinnung normativer Maßstäbe für eine gerechte Gesellschaftsordnung, begründet deren Inhalte, wirkt maßgeblich an deren Realisierung, dogmatischer Anwendung und kritischer Reflexion mit. Sie ist - trotz dieses Anspruches - indes nicht allein, sondern auf die Erkenntnisse anderer Wissenschaftsbereiche verwiesen. Notwendig sind insbesondere Erkenntnisse der Soziologie, der Psychologie und der ökonomischen Wissenschaften, um kritisch reflektieren und evaluieren zu können, ob die von der Rechtswissenschaft und vom demokratisch legitimierten Normgeber für richtig gehaltenen Rechtssätze ihr spezifisches Wirkungs- und Steuerungspotenzial auch wirklich entfalten (können). In Abwandlung eines bekannten Diktums: Rechtswissenschaft ohne Gerechtigkeit ist blind, gerechtes Recht ohne Wirksamkeit leer. Für ersteres kommt der Rechtswissenschaft ein maßgeblicher Legitimationsanspruch zu, für zweiteres ist sie auch auf die Erkenntnisse anderer Wissenschaften verwiesen. In einem so grundgelegten interdisziplinären Ansatz könnte ein beachtliches Forschungs- und Weiterentwicklungspotenzial der Rechtswissenschaft liegen - zum Wohle einer gerechten menschlichen Ordnung.

98 Vgl. dazu Lindner, Rechtswertungsfreier Raum (Fn. 70); ders., Tragische Konflikte, FAZ v. 15.10.2004, S. 8.

99 Vgl. etwa P.-A. Alt, Mehr Disziplin wagen, SZ v. 21.10.2010, S. 13: „Differenz entsteht durch Identität“. 